RESCEU-24/20

\title{
Baryon isocurvature constraints on the primordial hypermagnetic fields
}

\author{
Kohei Kamada, ${ }^{a}$ Fumio Uchida, ${ }^{a, b}$ and Jun'ichi Yokoyama ${ }^{a, b, c, d}$ \\ ${ }^{a}$ Research Center for the Early Universe (RESCEU), Graduate School of Science, \\ The University of Tokyo, Tokyo 113-0033, Japan \\ ${ }^{b}$ Department of Physics, Graduate School of Science, \\ The University of Tokyo, Tokyo 113-0033, Japan \\ ${ }^{c}$ Kavli Institute for the Physics and Mathematics of the Universe (Kavli IPMU), \\ WPI, UTIAS, The University of Tokyo, Kashiwa, Chiba 277-8568, Japan \\ ${ }^{d}$ Trans-scale Quantum Science Institute, \\ The University of Tokyo, Tokyo 113-0033, Japan \\ E-mail:kohei.kamada@resceu.s.u-tokyo.ac.jp, fuchida@resceu.s.u-tokyo.ac.jp, \\ yokoyama@resceu.s.u-tokyo.ac.jp
}

\begin{abstract}
It has been pointed out that hypermagnetic helicity decay at the electroweak symmetry breaking may have produced the observed baryon asymmetry of the Universe through the chiral anomaly in the standard model of particle physics. Although fully helical magnetic field that can adequately produce the observed baryon asymmetry is not strong enough to explain the origin of the intergalactic magnetic field inferred by the Fermi satellite, the mixture of helical and nonhelical primordial magnetic fields may explain both baryogenesis and the intergalactic magnetic fields simultaneously. We first show that such a scenario is ruled out by the constraint on the amplitude of baryon isocurvature perturbations produced by the primordial magnetic fields to avoid overproduction of deuterium at the big bang nucleosynthesis. Then we show that any attempt to explain the origin of intergalactic magnetic field by primordial magnetogenesis before the electroweak symmetry breaking does not work due to the above constraint irrespective of the helicity and baryogenesis mechanism.
\end{abstract}




\section{Contents}

1 Introduction 1

2 Baryogenesis from hypermangetic helicity decay 3

2.1 Generation of the net baryon asymmetry 4

2.2 Magnetic field spectrum 7

2.3 Baryon isocurvature perturbation 9

3 Observational constraints 10

3.1 Constraints from inhomogeneous Big Bang Nulceosynthesis 11

3.2 Magnetic field evolution until today and implications from the intergalactic magnetic fields

4 Specific models $\quad 15$

4.1 Delta function 16

4.2 A Power-law spectrum with an exponential cutoff 18

5 Baryon isocurvature constraints on non-helical hypermagnetic fields 21

6 Conclusion and Discussion 25

$\begin{array}{ll}\text { A Gauge choice } & 27\end{array}$

B Correlation functions in the position space 28

\section{Introduction}

The excess of baryons over anti-baryons in the present universe, or the baryon asymmetry of the universe (BAU), is a key ingredient in modern cosmology and essential for our very existence. It is quantified by the baryon-to-entropy ratio, precisely determined by the observation of the cosmic microwave background (CMB) [1] as $\eta_{B} \equiv n_{B} / s=(8.718 \pm 0.054) \times 10^{-11}$, which is in good agreement with the one required for the success of the Big Bang Nucleosynthesis (BBN) [2]. The Standard Model of particle physics (SM) is hard to explain this tiny but non-zero asymmetry, especially in the context of inflationary cosmology, and its origin has been regarded as a fundamental problem in particle physics and cosmology. A dynamical mechanism, baryogenesis, thus should have taken place in the early Universe after the end of inflation before the BBN, since inflation dilutes away the preexisting asymmetry.

To generate non-vanishing baryon asymmetry we must satisfy the Sakharov criteria [3]: baryon-number violation, $\mathrm{C}$ and $\mathrm{CP}$ violation, and departure from thermal equilibrium. Since they are hardly satisfied in the realms of the SM, new physical degrees of freedom beyond the $\mathrm{SM}$ are often introduced, e.g., new fields to make the electroweak symmetry breaking (EWSB) strongly first order and to give the source of CP violation for the electroweak baryogenesis [4], or the right-handed neutrinos for leptogenesis [5]. In this regard, a baryogenesis scenario from hypermagnetic helicity decay [6-11] is remarkable since it does not require any new degrees of freedom beyond the SM in the mechanism itself. It utilizes the chiral anomaly in the SM [12-14], which relates the changes in the global baryon (B) and lepton (L) number $\left(Q_{B}\right.$ and 
$\left.Q_{L}\right)$ to the changes in the $\mathrm{SU}(2)_{L}$ Chern-Simons number $\left(N_{\mathrm{CS}}^{L}\right)$ and the $\mathrm{U}(1)_{Y}$ hypermagnetic helicity $\left(\mathcal{H}_{Y}\right)$,

$$
\Delta Q_{B}=\Delta Q_{L}=N_{\mathrm{g}}\left(\Delta N_{\mathrm{CS}}^{L}-\frac{g^{\prime 2}}{16 \pi^{2}} \Delta \mathcal{H}_{Y}\right)
$$

where $N_{\mathrm{g}}=3$ is the number of fermion generations and $g^{\prime}$ is the gauge coupling for the $\mathrm{U}(1)_{Y}$ hypergauge interaction. Thus baryon asymmetry can be generated if the hypermagnetic helicity decay occurs. This idea can be realized in realistic cosmological scenarios such as the axion inflation [15-17], the SU(5) Grand Unified Theory baryogenesis [18-20] through the chiral plasma instability [21-23], or the Affleck-Dine mechanism [24, 25], in which the long-range $\mathrm{U}(1)_{Y}$ hypermagnetic fields are generated before the EWSB with helicity (See, e.g., Refs. [26-33]). The hypermagnetic helicity decay occurs mainly at the EWSB when the hypermagnetic fields are converted into the $\mathrm{U}(1)_{\mathrm{EM}}$ magnetic fields. The Sakharov criteria are satisfied by the SM chiral anomaly (B-violation) and the existence of the long-range helical hypermagnetic fields ( $\mathrm{C}$ and $\mathrm{CP}$-violation as well as the deviation from thermal equilibrium). It has been shown that in the electroweak crossover suggested by the $125 \mathrm{GeV}$ Higgs [34, 35] baryon asymmetry can remain until today [11] against the washout by the electroweak sphalerons [4]. In this scenario, the physics beyond the SM is required for magnetogenesis but not for baryogenesis.

An advantage of this scenario is that the $\mathrm{U}(1)_{Y}$ hypermagnetic fields that are responsible for baryogenesis can persist until the present time after the conversion into the U(1) EM magnetic fields at the EWSB as the intergalactic magnetic fields (IGMFs), which would act as its potential probe. Indeed, the existence of IGMFs has been suggested by the recent $\mathrm{TeV}$ blazar observations with Fermi-LAT [36-45]. The lack of secondary GeV photon in the blazar spectra, expected to be generated during the propagation in the intergalactic void, can be attributed to the IGMFs so that the lower bound of their strength and coherence length are given. If further observation of the IGMFs can pin down their strength and coherence length as well as their helicity, in principle we can test the scenario, which gives us insights into the origin of the IGMFs as well as that of BAU.

Previous studies have already shown that maximally helical $\mathrm{U}(1)_{Y}$ gauge fields that can explain the observationally suggested IGMF would suffer from baryon overproduction [11], unless the real transition from the hypermagnetic fields to the electromagnetic fields at the electroweak crossover is much more rapid than the one extrapolated from the result of the lattice calculation [34, 35]. One way to accommodate the baryogenesis and IGMFs indicated by the blazar observations is to take the $\mathrm{U}(1)_{Y}$ hypermagnetic fields to be partially helical, which might be realized by, e.g., an incomplete cancellation of the hypermagnetic helicity and the chiral asymmetry carried by the right-handed electrons generated in axion inflation via the chiral plasma instability [30]. ${ }^{1}$ In such a case, the baryogenesis is less effective so that stronger hyoermagnetic fields, possibly up to the ones consistent with the lower bound of the IGMFs, are needed to explain the present BAU. The purpose of this paper is to address further this option.

The key ingredient to investigate this situation but not to have been explored in depth yet is the baryon isocurvature perturbation, which should be generated at the scale corresponding to the coherence length of the hypermagnetic fields. It is well known that the

\footnotetext{
${ }^{1}$ Another option is to consider the second magnetogenesis after the EWSB. In this case we do not have direct relationship between the BAU and IGMFs.
} 
baryon isocurvature perturbations are constrained by the observations of cosmic microwave background (CMB) on large scales $\left(k \lesssim 0.1 \mathrm{Mpc}^{-1} ; k\right.$ is the comoving wavenumber) [46], which would give constraints on acausally generated hypermagnetic fields that never entered the hydrodynamic turbulence due to sufficiently large coherence length. ${ }^{2}$ The hypermagnetic fields with smaller coherence length, e.g., the ones that have once entered the hydrodynamic turbulence regime, do not receive stringent constraints from such cosmological observations. However, such small-scale baryon isocurvature perturbations may spoil the successful BBN and their spectrum can be constrained by the studies of inhomogeneous BBN [49-51]. In particular, it has recently been pointed out that the baryon isocurvature perturbation at a scale larger than the neutron diffusion scale at the BBN epoch, $k \lesssim 4 \times 10^{8} \mathrm{Mpc}^{-1}$, is constrained by the deuterium overproduction due to the second-order effect [51]. As a result, one can obtain an upper bound on their coherence length. We find that since the baryon isocurvature perturbation is generated even from non-helical hypermagnetic fields [6, 7], the constraint becomes severer for less helical hypermagnetic fields, in which case hypermagnetic fields with longer coherence length are needed for the BAU. Consequently, together with the constraint on their properties from the magnetohydrodynamics (MHD) [52], we find a lower bound of the helicity fraction, $\epsilon$, which gives an upper bound of the hypermagnetic field strength that can generate the present BAU. In terms of the present IGMF properties, the corresponding upper bound lies below the most stringent lower bound of the IGMF strength [44], independent of the detail of their properties. Moreover, since the non-helical part of the hypermagnetic fields contributes to the baryon isocurvature perturbation, it constrains their properties even in the case the homogeneous part of the BAU is generated by another mechanism. It turns out that regardless of their helicity properties, any hypermagnetic fields with too large strength and coherence length are not allowed before the EWSB. We conclude that the IGMFs suggested by the observation of the Fermi-LAT collaboration must have been generated after the EWSB so as not to undermine the success of the BBN, irrespective of their helical property as well as the generation mechanism of the present BAU. The baryogenesis from the hypermagnetic helicity decay can still be responsible for the present BAU, but we need additional magnetogenesis or an unknown mechanism of the magnetic field amplification after the EWSB.

The paper is organized as follows. In $\S 2$, we briefly review baryogenesis from hypermagnetic helicity decay and derive the spectrum of baryon isocurvature perturbation. We provide a general treatment with which we can assess whether this scenario survives against the baryon isocurvature constraints. In $\S 3$, we present the general formalism to constrain the scenario from baryon isocurvature perturbation and the way to connect the constraint to the IGMF observations, by taking into account the evolution of the magnetic fields after the EWSB. In $\S 4$, by adopting simple forms of the magnetic field power spectrum, we obtain constraints on the magnetic fields quantitatively in the case they are responsible for the present BAU. In $\S 5$, we present the constraints on the non-helical hypermagnetic fields without specifying baryogenesis models. Finally $\S 6$ is devoted to conclusion and discussion.

\section{Baryogenesis from hypermangetic helicity decay}

We begin by reviewing the baryogenesis scenario from the hypermagnetic helicity decay. In the previous studies [9-11] the hypermagnetic fields are parameterized by their characteristic

\footnotetext{
${ }^{2}$ The baryon isocurvature perturbations are degenerate with cold dark matter isocurvature perturbations. It has been claimed that $21 \mathrm{~cm}$ fluctuations can potentially discriminate the degeneracy [47], but still it is difficult to distinguish tiny isocurvature fluctuations [48].
} 
field strength $B_{\mathrm{p}}$ and coherence length $\xi_{\mathrm{p}}$, assuming a delta-function-like spectrum. For our purpose to investigate the baryon isocurvature perturbation, we here reformulate the consequence of this scenario by taking into account the spectrum of the hypermagnetic fields, as is also done in Ref. [6, 7].

\subsection{Generation of the net baryon asymmetry}

Let us first give the explanation how the net baryon asymmetry is generated by the decaying hypermagnetic helicity in a realistic cosmic history following Ref. [11]. As explained in the introduction, the change in the comoving hypermagnetic helicity density induces the change in the BAU through the chiral anomaly of the SM (Eq. (1.1)). Here we consider the case where the hypermagnetic fields are generated before the EWSB with sufficiently large coherence length and non-vanishing net helicity. Then the hypermagnetic fields evolve according to MHD. Basically, the net hypermagnetic helicity is almost conserved with the help of large electric conductivity [53,54] until the EWSB. However, the hypermagnetic helicity decay occurs in two ways in the cosmic history. The first is that the magnetic field diffusion due to the large but finite electric conductivity [6, 7], and the other is the electroweak symmetry breaking where the hypermagnetic helicity is converted into the $\mathrm{U}(1)_{\mathrm{EM}}$ magnetic helicity [11]. It turned out that the latter gives more significant contributions on the BAU, unless the dynamics of the EWSB is significantly different from the results of the lattice simulations [34, $35]$ as well as the one-loop analytic estimate [55]. Note that after the completion of the EWSB $\mathrm{U}(1)$ baryon symmetry is no longer anomalous and baryon asymmetry is not generated from the $\mathrm{U}(1)_{\mathrm{EM}}$ magnetic helicity decay. Below, we explain in more depth how the BAU is generated and how we can evaluate it. We here assume that the possible cancellation of the chiral asymmetry and hypermagnetic helicity [30] does not occur and the hypermagnetic helicity is much larger than the chiral asymmetry in the system before the EWSB.

To evaluate the BAU generated in this mechanism, we need to take into account the washout effects by the electroweak sphalerons [4], since this mechanism generates only $B+L$ but not $B-L$ asymmetry. If the EWSB would have completed earlier than the freezeout of the electroweak sphalerons, the baryon asymmetry would not remain much (See, however, Ref. [10]). However, lattice simulations of the EWSB in the light of the $125 \mathrm{GeV}$ Higgs suggest that they occur at almost the same time so that the electroweak sphaleron washout is incomplete. Then the evolution of the mean baryon asymmetry can be evaluated by solving a simplified kinetic equation for the mean baryon-to-entropy ratio, $\bar{\eta}_{B}$ [11],

$$
\frac{d \bar{\eta}_{B}}{d x}=(\text { source })-(\text { washout })
$$

with the dominant contributions around the EWSB $\left(T \simeq 130-145^{3} \mathrm{GeV}\right)$ being [11]

$$
\text { (source) }=\frac{3}{2}\left(g^{2}+g^{\prime 2}\right) \frac{d \theta_{\mathrm{w}}}{d \ln x} \sin 2 \theta_{\mathrm{w}} \mathcal{S}_{\mathrm{AB}}, \quad(\text { washout })=\frac{111}{34} \gamma_{\mathrm{w}, \mathrm{sph}} \bar{\eta}_{B},
$$

where

$$
\mathcal{S}_{\mathrm{AB}}=\frac{H}{8 \pi^{2} a^{3} s T} \overline{\boldsymbol{A}_{\mathcal{A}} \cdot \boldsymbol{B}_{\mathcal{A}}}
$$

with the overline representing the volume average. Here $x=M_{0} / T$ with $M_{0} \equiv M_{\mathrm{pl}} / \sqrt{\pi^{2} g_{*} / 90}$ $\left(M_{\mathrm{pl}} \simeq 2.43 \times 10^{18} \mathrm{GeV}\right.$ is the reduced Planck mass and $g_{*}$ is the effective number of relativistic degrees of freedom during the process.) and $T$ being the temperature of the Universe. $g$ is

\footnotetext{
${ }^{3}$ Electron Yukawa is dominant for the washout at $T \gtrsim 145 \mathrm{GeV}$.
} 
the gauge coupling for the $\mathrm{SU}(2)_{L}$ electroweak gauge interaction and $\theta_{\mathrm{w}}$ is the temperaturedependent effective weak mixing angle, which gradually changes from 0 to $\theta_{\mathrm{w} 0} \equiv \tan ^{-1}\left(g^{\prime} / g\right)$ at the electroweak crossover. $\gamma_{\mathrm{w}, \mathrm{sph}} \simeq \exp [-(146.6 \pm 1.0)+(0.83 \pm 0.01) \mathrm{T} / \mathrm{GeV}][34]$ is the transport coefficient for the electroweak sphalerons. $H \equiv \dot{a} / a$ is the Hubble parameter with $a$ being the scale factor and $s=\left(2 \pi^{2} / 45\right) g_{* s} T^{3}$ is the entropy density $\left(g_{* s}\right.$ is the relativistic degrees of freedom for entropy). Here $\mathcal{A}$ stands for the massless $\mathrm{U}(1)$ gauge field during the electroweak crossover, and $\boldsymbol{A}_{\mathcal{A}}$ and $\boldsymbol{B}_{\mathcal{A}}$ are the vector potential and comoving magnetic fields for $\mathcal{A}$, respectively. They are characterized by the effective weak mixing angle $\theta_{\mathrm{w}}$ as $\boldsymbol{Y}=\cos \theta_{\mathrm{w}} \boldsymbol{A}_{\mathcal{A}}$ and $\boldsymbol{W}^{3}=\sin \theta_{\mathrm{w}} \boldsymbol{A}_{\mathcal{A}}$, with $\boldsymbol{Y}$ and $\boldsymbol{W}^{3}$ being the vector potentials for the $\mathrm{U}(1)_{Y}$ hyper gauge interaction and the third component of the $\mathrm{SU}(2)_{L}$ electroweak gauge interaction. Here the kinetic equation is derived by taking into account the equilibrium conditions of the rapid spectator processes such as the spin-flipping process of chiral fermions through the Yukawa interactions other than the one for the electrons and the strong sphalerons. Note that during the electroweak crossover, the long-range "magnetic" field turns from that of $\mathrm{U}(1)_{Y}$ hypergauge interaction to that of the $\mathrm{U}(1)_{\mathrm{EW}}$ electromagnetic interaction through the massless $\mathrm{U}(1)$ gauge field $\mathcal{A}$. Thus the right hand side of the anomaly equation, $\left(g^{2} / 16 \pi^{2}\right) \operatorname{Tr}\left[W_{\mu \nu} \tilde{W}^{\mu \nu}\right]-\left(g^{\prime 2} / 32 \pi^{2}\right) Y_{\mu \nu} \tilde{Y}^{\mu \nu}$, includes the terms, e.g. $d\left(\sin \theta_{\mathrm{w}} \boldsymbol{A}_{\mathcal{A}}\right) / d \ln x \cdot \boldsymbol{B}_{\mathcal{A}} \sim\left(d \theta_{\mathrm{w}} / d \ln x\right) \boldsymbol{A}_{\mathcal{A}} \cdot \boldsymbol{B}_{\mathcal{A}} \cdot{ }^{4}$. The gradual changes of the weak mixing angle during the electroweak crossover give significant contributions to the source term. Around the electroweak symmetry breaking, the electroweak sphaleron becomes less and less effective and turns to the rate-determining reaction.

When the transport coefficient of the electroweak sphalerons is larger than the Hubble rate, the system enters the equilibrium state with $d \bar{\eta}_{B} / d x=0$ for Eq. (2.1), which gives the mean baryon asymmetry as

$$
\bar{\eta}_{B} \simeq \frac{17}{37} \frac{g^{2}+g^{\prime 2}}{\gamma_{\mathrm{w}, \mathrm{sph}}} \frac{d \theta_{\mathrm{w}}}{d \ln x} \sin 2 \theta_{\mathrm{w}} \mathcal{S}_{\mathrm{AB}}
$$

before the electroweak sphaleron freezeout. As the temperature of the Universe decreases, the electroweak sphaleron freezes out around $T=T_{\mathrm{fo}} \simeq 135 \mathrm{GeV}$. (Hereafter the subscript fo represents that the quantity is evaluated at the sphaleron freeze out, $T=T_{\text {fo }}$.) Just after the sphaleron freezeout the electroweak symmetry breaking completes and there will be no longer significant induction of baryon asymmetry. Numerically it is found that the resultant baryon asymmetry is evaluated the equilibrium solution (2.4) at $T_{\mathrm{fo}} \simeq 135 \mathrm{GeV}$ [11]. Since at the electroweak sphaleron freezeout, the transport coefficient of the electroweak sphaleron is related to the Hubble parameter as $\gamma_{\mathrm{w}, \mathrm{sph}}\left(T_{\mathrm{fo}}\right) \simeq H\left(T_{\mathrm{fo}}\right) / T_{\mathrm{fo}}$, the resultant baryon asymmetry is evaluated in a simplified form as

$$
\bar{\eta}_{B}=\left.\frac{17}{296 \pi^{2}}\left(g^{2}+g^{\prime 2}\right) \frac{d \theta_{\mathrm{w}}}{d \ln x} \sin 2 \theta_{\mathrm{w}} \frac{\overline{\boldsymbol{A}_{\mathcal{A}} \cdot \boldsymbol{B}_{\mathcal{A}}}}{a^{3} s}\right|_{T=T_{\mathrm{fo}}} .
$$

Therefore, if we have non-vanishing $\overline{\boldsymbol{A}_{\mathcal{A}} \cdot \boldsymbol{B}_{\mathcal{A}}}$ on average, we will obtain non-vanishing net baryon asymmetry. For a peaky spectrum of the hypermagnetic fields with the characteristic comoving field strength $B_{\mathrm{c}}$ (or the physical strength $B_{\mathrm{p}}=a^{-2} B_{\mathrm{c}}$ ) and the comoving coherence length $\xi_{\mathrm{c}}$ (or the physical coherence length $\xi_{\mathrm{p}}=a \xi_{\mathrm{c}}$ ) with the helicity fraction $\epsilon$, we can write

\footnotetext{
${ }^{4}$ We carry out all the computation with the Coulomb gauge $\boldsymbol{\nabla} \cdot \boldsymbol{A}_{\mathcal{A}}=0$, but the final results are gauge independent [11]
} 
$\overline{\boldsymbol{A}_{\mathcal{A}} \cdot \boldsymbol{B}_{\mathcal{A}}} \simeq \epsilon \xi_{\mathrm{c}} B_{\mathrm{c}}^{2}=a^{3} \epsilon \xi_{\mathrm{p}} B_{\mathrm{p}}^{2}$ and evaluate the net baryon asymmetry as

$$
\begin{aligned}
\bar{\eta}_{B} & =\left.\frac{17}{296 \pi^{2}}\left(g^{2}+g^{\prime 2}\right) \frac{d \theta_{\mathrm{w}}}{d \ln x} \sin 2 \theta_{\mathrm{w}} \frac{\epsilon \xi_{\mathrm{p}} B_{\mathrm{p}}^{2}}{s}\right|_{T=T_{\mathrm{fo}}} \\
& \left.\sim 10^{-10} \epsilon \sin 2 \theta_{\mathrm{w}} \frac{d \theta_{\mathrm{w}}}{d \ln x}\left(\frac{\xi_{\mathrm{p}}}{10^{6} \mathrm{GeV}^{-1}}\right)\left(\frac{B_{\mathrm{p}}}{10^{-3} \mathrm{GeV}^{2}}\right)^{2}\right|_{T=T_{\mathrm{fo}}},
\end{aligned}
$$

which will be more formally derived in the next subsection in terms of the magnetic field power spectrum.

The value of $\sin 2 \theta_{\mathrm{w}} d \theta_{\mathrm{w}} / d \ln x$ at $T=T_{\mathrm{fo}}$ has relatively large uncertainties in the analytic expressions [55] and results of the lattice calculations [35]. On the one hand, the time dependence of the weak mixing angle is evaluated analytically at the one-loop level as [55]

$$
\cos ^{2} \theta_{\mathrm{w}}=\cos ^{2} \theta_{\mathrm{w} 0}\left(1+\frac{11}{12} \frac{g_{3}^{2} \sin ^{2} \theta_{\mathrm{w} 0}}{\pi m_{W}(T)}\right)
$$

where $\theta_{\mathrm{w} 0}$ is the weak mixing angle at zero temperature, $g_{3}$ is the three dimensional $\mathrm{SU}(2)$ gauge coupling, and $m_{W}(T)$ is the perturbative $W$ boson mass. By substituting

$$
g_{3}^{2} \simeq g^{2} T, \quad m_{W}(T)=\frac{g \phi(T)}{2},
$$

with adopting the fitting function to the numerical lattice result for the Higgs condensate, $\phi(T)$, around the EWSB, $T \lesssim 162 \mathrm{GeV}[10]$,

$$
\phi(T) \simeq 0.23 T \sqrt{162-\frac{T}{1 \mathrm{GeV}}},
$$

we obtain

$$
\left.\frac{d \theta_{\mathrm{w}}}{d \ln x} \sin 2 \theta_{\mathrm{w}}\right|_{T=T_{\mathrm{fo}}}=-\left.T \frac{d \theta_{\mathrm{w}}}{d T} \sin 2 \theta_{\mathrm{w}}\right|_{T=T_{\mathrm{fo}}} \simeq 0.14
$$

where we have used $g=0.65$ and $\cos ^{2} \theta_{\mathrm{w} 0}=0.77$.

On the other hand, the three dimensional lattice calculation of the weak mixing angle has been calculated in the light of the $125 \mathrm{GeV}$ Higgs, for $140 \mathrm{GeV}<T<170 \mathrm{GeV}$ in Ref. [35]. In principle the lattice calculation is an all-orders calculation that includes even the non-perturbative effects. However, the result has relatively large errors and agrees with the analytic calculation only marginally. Since one cannot tell which estimate is more reliable and the analytic estimate is applicable only limited temperature ranges, which is not suitable for solving the kinetic equation numerically, Ref. [11] has adopted a phenomenological fitting formula with a smoothed step function for $\theta_{\mathrm{w}}$ as

$$
\cos ^{2} \theta_{\mathrm{w}}(T)=\cos ^{2} \theta_{\mathrm{w} 0}+\frac{1-\cos ^{2} \theta_{\mathrm{w} 0}}{2}\left(1+\tanh \frac{T-T_{\mathrm{step}}}{\Delta T}\right) .
$$

The values of the parameters $T_{\text {step }}$ and $\Delta T$, chosen in Ref. [11], which give relatively good fit for the lattice results are shown in Table 1.5 Once more, we do not yet have a definite

\footnotetext{
${ }^{5}$ The parameterization A in Ref. [11] is omitted since it does not give a good fit at $T<160 \mathrm{GeV}$ and Eq. (2.6) is not applicable for the estimate of the resultant baryon asymmetry [11].
} 


\begin{tabular}{|c|c|c|c|c|c|}
\hline parameterizations & B & $\mathrm{C}$ & $\mathrm{D}$ & $\mathrm{E}$ & one-loop analytic \\
\hline$T_{\text {step }} / \mathrm{GeV}$ & 160 & 160 & 155 & 155 & - \\
\hline$\Delta T / \mathrm{GeV}$ & 5 & 10 & 10 & 20 & - \\
\hline$-\left.T \frac{d \theta_{\mathrm{w}}}{d T} \sin 2 \theta_{\mathrm{w}}\right|_{T=T_{\mathrm{fo}}}$ & $6 \times 10^{-4}$ & $4 \times 10^{-2}$ & 0.1 & 0.3 & 0.14 \\
\hline
\end{tabular}

Table 1. Parameters that characterize the EWSB are shown. Parameterizations B-E are the ones with Eq. (2.11) chosen in Ref. [11], and the last column is the analytic estimate (Eq. (2.10)) with the the formula at the one-loop level in Ref. [55]. We use the latter as the fiducial value of the temperature dependence of the weak mixing angle for the estimate of the resultant baryon asymmetry.

answer for the estimate of the precise time dependence of the weak mixing angle and we admit typically $\mathcal{O}\left(10^{3}\right)$ uncertainty in the estimate of $d \theta_{\mathrm{w}} / d T$.

For the practical purpose, however, we adopt Eq. (2.10) as the fiducial value for the estimate of the baryon asymmetry, since the perturbative analytic estimate becomes more accurate at lower temperature $T<140 \mathrm{GeV}$, whereas the lattice calculation gives larger errors for lower temperature ${ }^{6}$. Combining Eqs. (2.6) and (2.10) (and even using Tab. 1), we can see that it is possible to explain the present BAU with appropriate properties of primordial hypermagnetic fields.

\subsection{Magnetic field spectrum}

To investigate the spatial distribution of the baryon asymmetry, it is clear from the right hand side of Eq. (2.5) that we need to know the spatial distribution of the magnetic fields. In this subsection, we provide the way to parameterize the spatial distributions of magnetic fields, namely, their power spectrum, by assuming that they act as stochastic fields, which is often realized in cosmic history from some magnetogenesis mechanisms such as those from axion inflation [15-17], standard inflation with a dilatonic coupling in the kinetic function of gauge fields [56-58], or the strong first order phase transitions [59-61]. In the next subsection, we provide the expressions of the baryon isocurvature perturbations in terms of the magnetic field power spectrum provided in this subsection. Here we consider that the magnetic field power spectrum smoothly converts from the one for the $\mathrm{U}(1)_{Y}$ hypergauge field and to the one for the $\mathrm{U}(1)_{\mathrm{EM}}$ electromagnetic field without significant decay or change of the scales since the change of the weak mixing angle is a relatively slow process during the electroweak crossover [35]. In what follows we do not distinguish the hypergauge field and the electromagnetic field, as well as the intermediate massless gauge field $\mathcal{A}$ and simply denote them as $A_{\mu}$ as long as the power spectrum is concerned.

Let us now study the distribution of the gauge field $A_{i}$ in the wavenumber space ${ }^{7}$ by adopting the Coulomb gauge (See the Appendix A for more explanation on the gauge fixing). The power spectrum of the vector potential is then defined as follows,

$$
\left\langle A_{i}^{*}(\boldsymbol{k}, t) A_{j}\left(\boldsymbol{k}^{\prime}, t\right)\right\rangle=(2 \pi)^{3} \delta^{3}\left(\boldsymbol{k}-\boldsymbol{k}^{\prime}\right) \mathcal{F}_{i j}^{A}(\boldsymbol{k}, t),
$$

with

$$
\mathcal{F}_{i j}^{A}(\boldsymbol{k}, t)=P_{i j}(\hat{\boldsymbol{k}}) S(k, t)+i \epsilon_{i j m} \hat{k}_{m} A(k, t),
$$

\footnotetext{
${ }^{6}$ We are grateful to Mikko Laine for pointing it out.

${ }^{7}$ We adopt $f(k)=\int d x e^{-i k x} f(x)$ and $f(x)=\int d k /(2 \pi) e^{i k x} f(k)$ as the Fourier transformation.
} 
where $\epsilon_{i j m}$ is the 3 -dimensional Levi-Civita tensor with $\epsilon_{123}=1$, and

$$
P_{i j}(\hat{\boldsymbol{k}}) \equiv \delta_{i j}-\hat{k}_{i} \hat{k}_{j}, \quad \hat{k}_{i} \equiv \frac{k_{i}}{k}, \quad k \equiv|\boldsymbol{k}| .
$$

$S(k, t)$ and $A(k, t)$ are the symmetric and anti-symmetric parts of the power spectrum of the magnetic fields, respectively. We keep them arbitrary for the moment to make our discussion general. Hereafter we omit the argument $t$ but implicitly assume the time dependence of these functions, which should obey the MHD. Assuming the Gaussianity of the magnetic field stochastic distribution, we can characterize all the properties of the magnetic fields solely by these two functions, $S(k)$ and $A(k)$, since the higher-order cumulants vanish for Gaussiandistributed stochastic variables. The comoving energy density, helicity density, and coherence length of the magnetic fields are now formally defined as

$$
\begin{aligned}
\mathcal{E}_{\mathrm{c}} & =\frac{1}{2 V} \int d^{3} x\left\langle\boldsymbol{B}(\boldsymbol{x})^{2}\right\rangle \\
& =\frac{1}{2} \int \frac{d^{3} k}{(2 \pi)^{3}} k^{2} \sum_{i} \mathcal{F}_{i i}^{A}(\boldsymbol{k})=\frac{1}{2 \pi^{2}} \int d k k^{4} S(k), \\
h_{\mathrm{c}} & =\frac{1}{V} \int d^{3} x\langle\boldsymbol{A}(\boldsymbol{x}) \cdot \boldsymbol{B}(\boldsymbol{x})\rangle \\
& =-i \epsilon_{i j m} \int \frac{d^{3} k}{(2 \pi)^{3}} k_{m} \mathcal{F}_{i j}^{A}(\boldsymbol{k})=\frac{1}{\pi^{2}} \int d k k^{3} A(k), \\
\xi_{\mathrm{c}} & =\frac{\int d k k^{3} S(k)}{\int d k k^{4} S(k)},
\end{aligned}
$$

respectively, where $V=\int d^{3} x$ is the volume factor. The helicity fraction $\epsilon$, which is in principle time-dependent, is defined as

$$
\epsilon \equiv \frac{h_{\mathrm{c}}}{2 \xi_{\mathrm{c}} \mathcal{E}_{\mathrm{c}}}
$$

Now from Eq. (2.5) the net mean baryon asymmetry is given in terms of the power spectrum as

$$
\begin{aligned}
\bar{\eta}_{B} & =\left.\frac{17}{296 \pi^{2}} \frac{g^{2}+g^{\prime 2}}{a^{3} s} \frac{d \theta_{\mathrm{w}}}{d \ln x} \sin 2 \theta_{\mathrm{w}} \frac{1}{\pi^{2}} \int d k k^{3} A(k)\right|_{T=T_{\mathrm{fo}}} \\
& \left.\equiv \mathcal{C} h_{\mathrm{c}}\right|_{T=T_{\mathrm{fo}}}=\left.2 \mathcal{C} \epsilon \xi_{\mathrm{c}} \mathcal{E}_{\mathrm{c}}\right|_{T=T_{\mathrm{fo}}},
\end{aligned}
$$

where we have defined

$$
\begin{aligned}
\mathcal{C} & \left.\equiv \frac{17}{296 \pi^{2}} \frac{g^{2}+g^{\prime 2}}{a^{3} s} \frac{d \theta_{\mathrm{w}}}{d \ln x} \sin 2 \theta_{\mathrm{w}}\right|_{T=T_{\mathrm{fo}}} \\
& \simeq 2 \times 10^{34} \mathrm{GeV}^{-3}\left(\frac{\left.\frac{d \theta_{\mathrm{w}}}{d \ln x} \sin 2 \theta_{\mathrm{w}}\right|_{T=T_{\mathrm{fo}}}}{0.14}\right) \simeq 1 \times 10^{33} \mathrm{Mpc}^{-1} \mathrm{G}^{-2}\left(\frac{\left.\frac{d \theta_{\mathrm{w}}}{d \ln x} \sin 2 \theta_{\mathrm{w}}\right|_{T=T_{\mathrm{fo}}}}{0.14}\right) .
\end{aligned}
$$

Here we have used $g^{\prime}=0.35, g=0.65, g_{* s}^{\text {fo }}=106.75$, and $a_{\mathrm{fo}}=5.8 \times 10^{-16}\left(\right.$ for $\left.T_{\mathrm{fo}}=135 \mathrm{GeV}\right)$. We have adopted the natural Heaviside-Lorentz units, ${ }^{8}$ whence $1 \mathrm{G}=1.95 \times 10^{-20} \mathrm{GeV}^{2}$ and $1 \mathrm{Mpc}=1.56 \times 10^{38} \mathrm{GeV}^{-1}$.

\footnotetext{
${ }^{8}$ In this unit system, $\hbar=c=\varepsilon_{0}=1$, while the natural Gaussian CGS units set $\hbar=c=4 \pi \varepsilon_{0}=1$.
} 
In the case when $S(k)$ is proportional to $A(k)$, the helicity fraction is found to be the proportional constant, $A(k)=\epsilon S(k)$. Note that the realizability condition [62] imposes $|\epsilon| \leq 1$. In particular, by requiring $\bar{\eta}_{B} \simeq 10^{-10}$, the following relation applies.

$$
\left(\frac{\left.\frac{d \theta_{\mathrm{w}}}{d \ln x} \sin 2 \theta_{\mathrm{w}}\right|_{T=T_{\mathrm{fo}}}}{0.14}\right)^{\frac{1}{2}}\left(\frac{\xi_{\mathrm{p}, \mathrm{fo}}}{10^{6} \mathrm{GeV}^{-1}}\right)^{\frac{1}{2}}\left(\frac{B_{\mathrm{p}, \mathrm{fo}}}{10^{-3} \mathrm{GeV}^{2}}\right) \simeq 5 \times \epsilon_{\mathrm{fo}}^{-\frac{1}{2}} .
$$

\subsection{Baryon isocurvature perturbation}

Now we are ready to express the baryon isocurvature perturbations in the wavenumber space, in terms of the magnetic field power spectrum. An expression in the position space is often useful as well. We explicitly derive it in Appendix B.

Equation (2.5) implies that the spatial distribution of the BAU at its freeze out, $\eta_{B, \text { fo }}$, is proportional to $\left.\boldsymbol{A} \cdot \boldsymbol{B}\right|_{T=T_{\mathrm{fo}}}$,

$$
\eta_{B, \mathrm{fo}}(\boldsymbol{x})=\left.\mathcal{C} \boldsymbol{A}(\boldsymbol{x}) \cdot \boldsymbol{B}(\boldsymbol{x})\right|_{T=T_{\mathrm{fo}}}=\left.\mathcal{C}(\boldsymbol{A} \cdot \boldsymbol{\nabla} \times \boldsymbol{A})(\boldsymbol{x})\right|_{T=T_{\mathrm{fo}}},
$$

whose Fourier mode is expressed as

$$
\eta_{B, \mathrm{fo}}(\boldsymbol{k}) \equiv \int d^{3} r e^{-i \boldsymbol{k} \cdot \boldsymbol{r}} \eta_{B, \mathrm{fo}_{\mathrm{o}}}(\boldsymbol{r})=i \epsilon_{i j k} \mathcal{C} \int \frac{d^{3} p}{(2 \pi)^{3}} A_{i}^{*}(\boldsymbol{p}-\boldsymbol{k}) p_{j} A_{k}(\boldsymbol{p}) .
$$

To investigate the distribution of the baryon-number fluctuation, $\delta \eta_{B}(\boldsymbol{x}) \equiv \eta_{B}(\boldsymbol{x})-\bar{\eta}_{B}$, we use two point function of the relative fluctuation, $S_{B}(\boldsymbol{x}) \equiv \delta \eta_{B}(\boldsymbol{x}) / \bar{\eta}_{B}$, expressed as

$$
\mathcal{G}(\boldsymbol{r}) \equiv\left\langle S_{B, \mathrm{fo}}(\boldsymbol{x}) S_{B, \mathrm{fo}}(\boldsymbol{x}+\boldsymbol{r})\right\rangle=\frac{\left\langle\eta_{B, \mathrm{fo}}(\boldsymbol{x}) \eta_{B, \mathrm{fo}}(\boldsymbol{x}+\boldsymbol{r})\right\rangle}{\bar{\eta}_{B}^{2}}-1 .
$$

Then its Fourier transform or the power spectrum is given by

$$
\begin{aligned}
\mathcal{G}(\boldsymbol{k}) & =\frac{1}{V} \frac{\left\langle\left|\eta_{B, \mathrm{fo}}(\boldsymbol{k})\right|^{2}\right\rangle}{\bar{\eta}_{B}^{2}}-(2 \pi)^{3} \delta^{3}(\boldsymbol{k}) \\
& =\epsilon_{i j k} \epsilon_{l m n} \frac{\mathcal{C}^{2}}{V \bar{\eta}_{B}^{2}} \int \frac{d^{3} p}{(2 \pi)^{3}} \int \frac{d^{3} p^{\prime}}{(2 \pi)^{3}} p_{j} p_{m}^{\prime} J_{i k l n}\left(\boldsymbol{k}-\boldsymbol{p}, \boldsymbol{p}, \boldsymbol{k}-\boldsymbol{p}^{\prime}, \boldsymbol{p}^{\prime}\right)-(2 \pi)^{3} \delta^{3}(\boldsymbol{k}),
\end{aligned}
$$

where

$$
\begin{aligned}
J_{i k l n}\left(\boldsymbol{q}_{1}, \boldsymbol{q}_{2}, \boldsymbol{q}_{3}, \boldsymbol{q}_{4}\right) \equiv & \left\langle A_{i}^{*}\left(\boldsymbol{q}_{1}\right) A_{k}^{*}\left(\boldsymbol{q}_{2}\right) A_{l}\left(\boldsymbol{q}_{3}\right) A_{n}\left(\boldsymbol{q}_{4}\right)\right\rangle \\
=\left\langle A_{i}^{*}\left(\boldsymbol{q}_{1}\right) A_{k}^{*}\left(\boldsymbol{q}_{2}\right)\right\rangle\left\langle A_{l}\left(\boldsymbol{q}_{3}\right) A_{n}\left(\boldsymbol{q}_{4}\right)\right\rangle & +\left\langle A_{i}^{*}\left(\boldsymbol{q}_{1}\right) A_{l}\left(\boldsymbol{q}_{3}\right)\right\rangle\left\langle A_{k}^{*}\left(\boldsymbol{q}_{2}\right) A_{n}\left(\boldsymbol{q}_{4}\right)\right\rangle \\
& +\left\langle A_{i}^{*}\left(\boldsymbol{q}_{1}\right) A_{n}\left(\boldsymbol{q}_{4}\right)\right\rangle\left\langle A_{k}^{*}\left(\boldsymbol{q}_{2}\right) A_{l}\left(\boldsymbol{q}_{3}\right)\right\rangle \\
=(2 \pi)^{6}\left[\delta ^ { 3 } \left(\boldsymbol{q}_{1}\right.\right. & \left.+\boldsymbol{q}_{2}\right) \delta^{3}\left(\boldsymbol{q}_{3}+\boldsymbol{q}_{4}\right) \mathcal{F}_{i k}^{A}\left(\boldsymbol{q}_{1}\right) \mathcal{F}_{l n}^{A}\left(-\boldsymbol{q}_{3}\right) \\
& +\delta^{3}\left(\boldsymbol{q}_{1}-\boldsymbol{q}_{3}\right) \delta^{3}\left(\boldsymbol{q}_{2}-\boldsymbol{q}_{4}\right) \mathcal{F}_{i l}^{A}\left(\boldsymbol{q}_{1}\right) \mathcal{F}_{k n}^{A}\left(\boldsymbol{q}_{2}\right) \\
& \left.+\delta^{3}\left(\boldsymbol{q}_{1}-\boldsymbol{q}_{4}\right) \delta^{3}\left(\boldsymbol{q}_{2}-\boldsymbol{q}_{3}\right) \mathcal{F}_{i n}^{A}\left(\boldsymbol{q}_{1}\right) \mathcal{F}_{k l}^{A}\left(\boldsymbol{q}_{2}\right)\right] .
\end{aligned}
$$

Here we have used the reality condition of the gauge fields, $A_{i}^{*}(\boldsymbol{k})=A_{i}(-\boldsymbol{k})$. In the second equality, we have decomposed the four-point function of the vector potential into pairs of 
two-point functions, assuming the Gaussian distribution. The contribution of each term to Eq. (2.25) is evaluated as follows. The first term in the last line of Eq. (2.26) is calculated as

$$
\begin{array}{r}
\epsilon_{i j k} \epsilon_{l m n} \frac{(2 \pi)^{6} \mathcal{C}^{2}}{V \bar{\eta}_{B}^{2}} \int \frac{d^{3} p}{(2 \pi)^{3}} \int \frac{d^{3} p^{\prime}}{(2 \pi)^{3}} p_{j} p_{m}^{\prime}\left[\delta^{3}(\boldsymbol{k})\right]^{2} \mathcal{F}_{i k}^{A}(\boldsymbol{k}-\boldsymbol{p}) \mathcal{F}_{l n}^{A}\left(\boldsymbol{p}^{\prime}-\boldsymbol{k}\right) \\
=\frac{(2 \pi)^{3} \mathcal{C}^{2}}{\bar{\eta}_{B}^{2}}\left[\frac{1}{\pi^{2}} \int d p p^{3} A(p)\right]^{2} \delta^{3}(\boldsymbol{k})=(2 \pi)^{3} \delta^{3}(\boldsymbol{k})
\end{array}
$$

This contribution is nothing but the homogeneous component of the baryon asymmetry and cancels with the last term in Eq. (2.25). It is generated only from the antisymmetric part of the power spectrum. On the other hand, the remaining contributions, which generate the inhomogeneities of the baryon asymmetry, come from both symmetric and antisymmetric parts. This can be seen by explicitly calculating the second term together with the third term as

$$
\begin{aligned}
\epsilon_{i j k} \epsilon_{l m n} \frac{(2 \pi)^{6} \mathcal{C}^{2}}{V \bar{\eta}_{B}^{2}} \int \frac{d^{3} p}{(2 \pi)^{3}} \int \frac{d^{3} p^{\prime}}{(2 \pi)^{3}} p_{j} p_{m}^{\prime} & \left\{\left[\delta^{3}\left(\boldsymbol{p}-\boldsymbol{p}^{\prime}\right)\right]^{2} \mathcal{F}_{i l}^{A}(\boldsymbol{k}-\boldsymbol{p}) \mathcal{F}_{k n}^{A}(\boldsymbol{p})\right. \\
+ & {\left.\left[\delta^{3}\left(\boldsymbol{k}-\boldsymbol{p}-\boldsymbol{p}^{\prime}\right)\right]^{2} \mathcal{F}_{i n}^{A}(\boldsymbol{k}-\boldsymbol{p}) \mathcal{F}_{k l}^{A}(\boldsymbol{p})\right\} } \\
=\frac{\mathcal{C}^{2}}{\bar{\eta}_{B}^{2}} \int \frac{d^{3} p}{(2 \pi)^{3}}\left[p^{2} S(|\boldsymbol{k}-\boldsymbol{p}|) S(p)+\right. & |\boldsymbol{k}-\boldsymbol{p}| p A(|\boldsymbol{k}-\boldsymbol{p}|) A(p)] \\
\times & {\left[1-\frac{2(\boldsymbol{k}-\boldsymbol{p}) \cdot \boldsymbol{p}}{p^{2}}+\frac{((\boldsymbol{k}-\boldsymbol{p}) \cdot \boldsymbol{p})^{2}}{|\boldsymbol{k}-\boldsymbol{p}|^{2} p^{2}}\right] . }
\end{aligned}
$$

Putting Eqs. (2.27) and (2.28) into Eq. (2.25), we obtain

$$
\begin{aligned}
\mathcal{G}(\boldsymbol{q})=\frac{\mathcal{C}^{2}}{\bar{\eta}_{B}^{2}} \int \frac{d^{3} p}{(2 \pi)^{3}}\left[p^{2} S(|\boldsymbol{k}-\boldsymbol{p}|)\right. & S(p)+|\boldsymbol{k}-\boldsymbol{p}| p A(|\boldsymbol{k}-\boldsymbol{p}|) A(p)] \\
\times & {\left[1-\frac{2(\boldsymbol{k}-\boldsymbol{p}) \cdot \boldsymbol{p}}{p^{2}}+\frac{((\boldsymbol{k}-\boldsymbol{p}) \cdot \boldsymbol{p})^{2}}{|\boldsymbol{k}-\boldsymbol{p}|^{2} p^{2}}\right] . }
\end{aligned}
$$

The essential point, which has already been noticed in Ref. [7], is that the non-helical part $S(k)$ contributes to the two-point correlation function of the magnetic helicity. This is because $A(k)=0$ does not mean that the helicity, $\boldsymbol{A} \cdot \boldsymbol{B}$, vanishes at every position for $S(k) \neq 0$. Thus the two-point correlation function of the magnetic helicity yields a non-vanishing value even for $A(k)=0$, which plays crucial role in constraining the primordial hypermagnetic fields.

\section{Observational constraints}

The baryon isocurvature perturbation, which we explored in the previous section, imposes constraints on the baryogenesis scenario from the hypermagnetic helicity decay, in the parameter space where the homogeneous part of the BAU can be explained. In this section, we examine these constraints in terms of the magnetic field properties. We also briefly summarize the implication of the observation of the intergalactic magnetic fields on the scenario, adopting the magnetic field evolution suggested by the MHD. 


\subsection{Constraints from inhomogeneous Big Bang Nulceosynthesis}

Let us first examine the constraint from the baryon isocurvature perturbations. It is wellknown that they are strongly constrained by the CMB [46, 63-66]. ${ }^{9}$ However, it is sensitive only to the comoving scales larger than $10 \mathrm{Mpc}$. In the scenario of the baryogenesis from hypermagnetic helicity decay, baryon isocurvature perturbations are produced on much smaller scales, since they correspond to the magnetic field coherence length as we have seen in the previous section, which depends on the magnetogenesis mechanism. Thus the constraints from the CMB observations are generally not applicable.

Fortunately, baryon isocurvature perturbation on much smaller scales can be constrained by the inhomogeneous BBN [51]. Relatively small-scale baryon number fluctuations, larger than the comoving neutron diffusion scale at the $\mathrm{BBN}, k_{\mathrm{d}}^{-1} \simeq 0.0025 \mathrm{pc}$, should not be very large; otherwise it changes the predictions of the primordial abundance of light elements and spoils the success of the BBN. Thus we can rule out too large baryon isocurvature perturbations at a smaller scale than the scales constrained by the CMB observations. In contrast, a large inhomogeneity on scales smaller than $k_{\mathrm{d}}^{-1}$ at the BBN can be smeared out by the neutron diffusion, and the constraints get weaker.

In our scenario, the typical scale of the baryon isocurvature perturbation is implemented in the magnetic field coherence length, characterized by $\xi_{\mathrm{c}}$, as we have seen in the previous section. Since at the scale $\sim \xi_{\mathrm{c}}$ the amplitude of the baryon isocurvature perturbation peaks to reach $\mathcal{O}(1)$ at the EWSB, scenarios with large coherence length, $\xi_{\mathrm{c}} \gg k_{\mathrm{d}}^{-1}$, would create antibaryon domains in the observable Universe at the BBN and are not allowed. Such a large coherence length is realized for the hypermagnetic fields produced in inflationary magnetogenesis or hypermagnetic fields in the MHD turbulence with relatively large field strength. On the contrary, for smaller coherence length, $\xi_{\mathrm{c}} \lesssim k_{\mathrm{d}}^{-1}$, baryon isocurvature perturbation at the BBN leads to the inhomogeneous BBN (IBBN), explored in Refs. [68-72]. In this case, neutrons diffuse and damp large baryon isocurvature perturbations at the scales smaller than the neutron diffusion scale, and there remains tiny but non-vanishing baryon isocurvature perturbations at the scales larger than the neutron diffusion scale, from which one can constrain the magnetic field distributions. By treating the baryon inhomogeneities at the BBN that survives the smearing effect of the neutron diffusion as a linear perturbation, one can estimate the deuterium abundance from the second order effect of perturbations to obtain a constraint on the baryon isocurvature perturbations to avoid the deutrium overproduction. The condition for the volume average of the baryon isocurvature perturbation is found to be [51]

$$
\overline{S_{B, \mathrm{BBN}}^{2}(\boldsymbol{x})}<0.016 \quad(2 \sigma),
$$

where $S_{B, \mathrm{BBN}}(\boldsymbol{x})$ is the smoothed baryon isocurvature perturbation at the BBN.

To take into account the smearing effect of the neutron diffusion, we introduce a Gaussian window function so that the baryon perturbation $\delta \eta_{B}$ at the $\mathrm{BBN}$ is evaluated as

$$
\delta \eta_{B, \mathrm{BBN}}(\boldsymbol{r})=\int d^{3} x W_{D}(\boldsymbol{x}-\boldsymbol{r}) \delta \eta_{B, \mathrm{fo}}(\boldsymbol{x}), \quad W_{D}(\boldsymbol{r}) \equiv\left(\frac{D}{\pi}\right)^{\frac{3}{2}} e^{-D \boldsymbol{r}^{2}}, \quad D \equiv \frac{3}{2} k_{\mathrm{d}}^{2} .
$$

The choice of the window function is motivated by the fact that the neutron diffusion equation is approximated by a heat equation [49] whose (three-dimensional) heat kernel is the Gaussian

\footnotetext{
${ }^{9}$ In addition, future $21 \mathrm{~cm}$ line observations are expected to yield constraints on the baryon isocurvature perturbations on smaller scales [47, 48, 67].
} 
function. Thus we employ $\eta_{B, \text { fo }}(\boldsymbol{r})$ as the initial condition and just convolute it with the heat kernel $W(\boldsymbol{r})$ to obtain $\eta_{B, \mathrm{BBN}}(\boldsymbol{r})$. The heat kernel is nothing but the window function, seen in the wavenumber space and is normalized as $\int d^{3} x W_{D}(\boldsymbol{x})=1$, or equivalently $W_{D}(\boldsymbol{k}=0)=1$ in the wavenumber space. We set the width of the Gaussian function so that the root mean square radius $\left(\int d^{3} x W_{D}(\boldsymbol{x}) x^{2}\right)^{1 / 2}=\sqrt{3 /(2 D)}$ coincides with $k_{\mathrm{d}}^{-1}$, obeying the definition of the word "diffusion length" in Ref. [49].

From Eq. (3.2), the volume average of the smoothed baryon isocurvature perturbation is evaluated as

$$
\begin{aligned}
\overline{S_{B, \mathrm{BBN}}^{2}} & =\frac{\left\langle\delta \eta_{B, \mathrm{BBN}}^{2}(\boldsymbol{x})\right\rangle}{\bar{\eta}_{B}^{2}}=\int d^{3} x W_{D / 2}(\boldsymbol{x}) \mathcal{G}(\boldsymbol{x}) \\
& =\int \frac{d^{3} k}{(2 \pi)^{3}} e^{-\frac{k^{2}}{2 D}} \mathcal{G}(\boldsymbol{k})(<0.016) .
\end{aligned}
$$

By inserting Eq. (2.29) into the last line, we obtain a formula that directly relates the power spectrum of the magnetic field to the baryon isocurvature perturbations at the BBN.

$$
\begin{aligned}
& \overline{S_{B, \mathrm{BBN}}^{2}}=\int \frac{d^{3} k}{(2 \pi)^{3}} e^{-\frac{k^{2}}{2 D}} \mathcal{G}(\boldsymbol{k}) \\
&=\frac{\mathcal{C}^{2}}{\bar{\eta}_{B}^{2}} \int \frac{d^{3} k}{(2 \pi)^{3}} \int \frac{d^{3} p}{(2 \pi)^{3}} e^{-\frac{k^{2}}{2 D}}\left[p^{2} S(|\boldsymbol{k}-\boldsymbol{p}|) S(p)+|\boldsymbol{k}-\boldsymbol{p}| p A(|\boldsymbol{k}-\boldsymbol{p}|) A(p)\right] \\
& \times\left[1-\frac{2(\boldsymbol{k}-\boldsymbol{p}) \cdot \boldsymbol{p}}{p^{2}}+\frac{((\boldsymbol{k}-\boldsymbol{p}) \cdot \boldsymbol{p})^{2}}{|\boldsymbol{k}-\boldsymbol{p}|^{2} p^{2}}\right] \\
&=\frac{\mathcal{C}^{2}}{4 \pi^{4} \bar{\eta}_{B}^{2}} \int d k_{1} d k_{2} k_{1}^{2} k_{2}^{2} \sum_{ \pm}\left( \pm\left\{\frac{\left(k_{1} \pm k_{2}\right)^{2}}{2}\left[S\left(k_{1}\right) S\left(k_{2}\right) \pm A\left(k_{1}\right) A\left(k_{2}\right)\right] \frac{D}{k_{1} k_{2}}\left(1 \mp \frac{D}{k_{1} k_{2}}\right)\right.\right. \\
&\left.\left.+\left[\frac{k_{1}^{2}+k_{2}^{2}}{2} S\left(k_{1}\right) S\left(k_{2}\right)+k_{1} k_{2} A\left(k_{1}\right) A\left(k_{2}\right)\right]\left(\frac{D}{k_{1} k_{2}}\right)^{3}\right\} \exp \left[-\frac{\left(k_{1} \mp k_{2}\right)^{2}}{2 D}\right]\right) .
\end{aligned}
$$

For a given magnetic field spectra, $A(k)$ and $S(k)$, with Eqs. (3.1) and (3.5) one can determine the constraints on their parameters, which is the main result of the present paper. Here we do not assume that the homogeneous part of the baryon asymmetry, $\bar{\eta}_{B}$, is produced by the hypermagnetic helicity decay. Therefore, we can adopt Eq. (3.5) also for the case where other baryogenesis mechanisms are responsible for the net BAU, which will be investigated in $\S 5$.

We also note that there is another issue at the BBN on the constraint of the magnetic field properties. Mainly because magnetic fields contribute to the energy density of the Universe as an additional relativistic degree of freedom at the time of the BBN, which are constrained in terms of the effective numbers of neutrino species, the magnetic field energy density or the field strength is constrained as $B_{\mathrm{c}, \mathrm{BBN}} \lesssim 10^{-6} \mathrm{G}$ [73] irrespective to its coherent length.

\subsection{Magnetic field evolution until today and implications from the intergalactic magnetic fields}

The long-range (hyper)magnetic fields generated in the radiation dominated era (including before the EWSB) will evolve according to the MHD equations and can remain until today as the IGMFs. Thus, the constraints obtained in the way we developed in the previous subsection 
can be rewritten in terms of the parameters of the IGMFs. In this subsection, we give the formulae that connect the properties of the magnetic fields at the EWSB and the present.

With sufficiently strong magnetic fields, the bulk velocity fields are excited due to the MHD, and the system enters the turbulence regime once the eddy turnover scale of the velocity fields catches up the coherence length of the magnetic fields. Before that the magnetic fields evolve adiabatically (the spectrum is unchanged in terms of the comoving quantities) whereas they evolve according to the scaling law of the direct or inverse cascade process until the recombination [52]. We assume that the magnetic fields evolve adiabatically again after the recombination.

More concretely, we treat the magnetic field evolution as follows. At the EWSB the coherence length of the magnetic fields should be longer than the eddy turnover scale [52, 74],

$$
\left.\xi_{\mathrm{p}}\right|_{T=T_{\mathrm{fo}}}>\left.\left.\xi_{\mathrm{p}, \mathrm{ed}}\right|_{T=T_{\mathrm{fo}}} \simeq v_{A}\right|_{T=T_{\mathrm{fo}}} t_{\mathrm{fo}}=\left.\left(\frac{2 \mathcal{E}_{\mathrm{p}}}{\rho_{\mathrm{p}}+p_{\mathrm{p}}}\right)^{\frac{1}{2}}\right|_{T=T_{\mathrm{fo}}} t_{\mathrm{fo}},
$$

where $\rho_{\mathrm{p}}$ and $p_{\mathrm{p}}$ are the physical energy density and pressure of the charged plasma particles, respectively, and the subscript $\mathrm{p}$ represents that the quantity is the physical one. Here we have assumed that the fluid velocity reaches at the Alfven velocity $v_{A}=\sqrt{2 \mathcal{E}_{\mathrm{p}} /\left(\rho_{\mathrm{p}}+p_{\mathrm{p}}\right)}=$ $\sqrt{2 \mathcal{E}_{\mathrm{c}} /\left(\rho_{\mathrm{c}}+p_{\mathrm{c}}\right)}$, which indicates the equipartition between the magnetic fields and fluid velocity fields. Note that it is convenient to investigate with physical quantities. If $\left.\xi_{\mathrm{p}}\right|_{T=T_{\mathrm{fo}}}=$ $\left.\xi_{\mathrm{p}, \mathrm{ed}}\right|_{T=T_{\mathrm{fo}}}$, this suggests that the magnetic fields have already started to evolve according to the cascade process at the EWSB. If the coherence length is longer than the eddy turnover scale at the EWSB, eventually the eddy turnover scale catches up the coherence length of the magnetic fields at

$$
t=\left.t_{\mathrm{sc}} \equiv \frac{\xi_{\mathrm{p}}}{\sqrt{2 \mathcal{E}_{\mathrm{p}} /\left(\rho_{\mathrm{p}}+p_{\mathrm{p}}\right)}}\right|_{t=t_{\mathrm{sc}}},
$$

and the cascade process is started. Note that if $t_{\mathrm{rec}}<t_{\mathrm{sc}}$, with $t_{\mathrm{rec}}$ being the physical cosmic time at the recombunation, the system never enters the cascade regime and evolve always adiabatically in the entire cosmic history after magnetogenesis.

The scaling law of the cascade process of the magnetic field evolution depends on the complicated properties of the distributions of the magnetic fields as well as the fluid velocities, which is often hard to determine. Instead of identifying the scaling law with such properties, with turning back to the comoving quantities, let us parameterize the scaling evolution of the magnetic fields, following Ref. [75], as

$$
\mathcal{E}_{\mathrm{c}} \propto \tau^{-\frac{2(\beta+1)}{\beta+3}}, \quad \xi_{\mathrm{c}} \propto \tau^{\frac{2}{\beta+3}},
$$

where $\tau$ is the conformal time, and $\beta$ is a parameter determined by the initial conditions of the magnetic fields and the fluid velocity fields. Note however that the magnetic field spectrum alone does not fix the value of $\beta .{ }^{10}$ Such a scaling law has also been considered in Refs. [76, 77]. The eddy turnover scale evolves with $\xi_{\mathrm{p}, \text { ed }}=t \sqrt{2 \mathcal{E}_{\mathrm{c}}(\tau) /\left(\rho_{\mathrm{c}}+p_{\mathrm{c}}\right)} \propto \tau^{2 /(\beta+3)}$ in the radiation dominated Universe and hence the relationship $\xi \simeq \xi_{\text {ed }}$ is continuously satisfied during the cascade regime. If the magnetic fields are maximally helical, the helicity conservation uniquely

\footnotetext{
${ }^{10} \beta$ is the parameter that quantifies the decay of the spectral energy around the comoving wave number $k=\xi_{c}^{-1}[75]$. It is an independent parameter of $\alpha$ in $\S 4.2$, which parameterizes the shape of the magnetic field power spectrum.
} 
determines the scaling law of the magnetic field evolution as $\beta=0$, which corresponds to the so-called "inverse cascade" regime. On the other hand, if the magnetic fields are not maximally helical, there are not apparent conserved quantities to determine $\beta$, and hence we here take $\beta(\geq 0)$ as the parameter of the system but do not explore its physical origin. In particular, we take $\beta=2$ as a representative value for the non-maximally helical case, which is often realized in the numerical simulations in Ref. [77]. We keep in mind that a smaller $\beta$ is hardly realized. For partially helical magnetic fields with the helicity fraction less than unity at the EWSB, $\left|\epsilon_{\mathrm{fo}}\right|<1$, the system becomes more and more helical with the help of the helicity conservation, and the helicity fraction $\epsilon$ grows through the cascade process [78], $\epsilon \propto \tau^{2 \beta /(\beta+3)}$. If $\epsilon_{\mathrm{fo}}>\left(\tau_{\mathrm{rec}} / \tau_{\mathrm{sc}}\right)^{-2 \beta /(\beta+3)}$, the system becomes maximally helical, $\epsilon=1$, before the recombination at $\tau=\tau_{\mathrm{sc}} \epsilon_{\mathrm{fo}}^{-(\beta+3) / 2 \beta}$, and $\beta$ turns to 0 . If $\epsilon_{\mathrm{fo}}<\left(\tau_{\mathrm{rec}} / \tau_{\mathrm{sc}}\right)^{-2 \beta /(\beta+3)}$, the cascade process is described by the single $\beta$ throughout until the recombination as long as the helicity fraction is less than 1 . Assuming that the magnetic field evolve adiabatically after the recombination until the present, we can express the present magnetic field energy density $\mathcal{E}_{0}$ and coherence length $\xi_{0}$ as

$$
\begin{aligned}
& \mathcal{E}_{0}= \begin{cases}\left.\epsilon_{\mathrm{fo}}^{\frac{2}{3}}\left(\frac{\tau_{\mathrm{sc}}}{\tau_{\mathrm{rec}}}\right)^{\frac{2}{3}} \mathcal{E}_{\mathrm{c}}\right|_{T=T_{\mathrm{fo}}} \simeq 1.1 \times\left. 10^{-8} \epsilon_{\mathrm{fo}}^{\frac{2}{3}}\left(\frac{\tau_{\mathrm{sc}}}{\tau_{\mathrm{fo}}}\right)^{\frac{2}{3}} \mathcal{E}_{\mathrm{c}}\right|_{T=T_{\mathrm{fo}}} & \text { for } \quad \epsilon_{\mathrm{fo}}>\left(\frac{\tau_{\mathrm{rec}}}{\tau_{\mathrm{sc}}}\right)^{-\frac{2 \beta}{\beta+3}}, \\
\left.\left(\frac{\tau_{\mathrm{sc}}}{\tau_{\mathrm{rec}}}\right)^{\frac{2(\beta+1)}{\beta+3}} \mathcal{E}_{\mathrm{c}}\right|_{T=T_{\mathrm{fo}}} & \text { for } \quad \epsilon_{\mathrm{fo}}<\left(\frac{\tau_{\mathrm{rec}}}{\tau_{\mathrm{sc}}}\right)^{-\frac{2 \beta}{\beta+3}},\end{cases} \\
& \xi_{0}= \begin{cases}\left.\epsilon_{\mathrm{fo}}^{\frac{1}{3}}\left(\frac{\tau_{\mathrm{rec}}}{\tau_{\mathrm{sc}}}\right)^{\frac{2}{3}} \xi_{\mathrm{c}}\right|_{T=T_{\mathrm{fo}}} \simeq 9.3 \times\left. 10^{7} \epsilon_{\mathrm{fo}}^{\frac{1}{3}}\left(\frac{\tau_{\mathrm{fo}}}{\tau_{\mathrm{sc}}}\right)^{\frac{2}{3}} \xi_{\mathrm{c}}\right|_{T=T_{\mathrm{fo}}} & \text { for } \epsilon_{\mathrm{fo}}>\left(\frac{\tau_{\mathrm{rec}}}{\tau_{\mathrm{sc}}}\right)^{-\frac{2 \beta}{\beta+3}} \\
\left.\left(\frac{\tau_{\mathrm{rec}}}{\tau_{\mathrm{sc}}}\right)^{\frac{2}{\beta+3}} \xi_{\mathrm{c}}\right|_{T=T_{\mathrm{fo}}} & \text { for } \epsilon_{\mathrm{fo}}<\left(\frac{\tau_{\mathrm{rec}}}{\tau_{\mathrm{sc}}}\right)^{-\frac{2 \beta}{\beta+3}}\end{cases}
\end{aligned}
$$

where we have used $\tau_{\text {rec }} / \tau_{\text {fo }}=\left(\tau_{\text {rec }} / \tau_{\text {eq }}\right) \cdot\left(\tau_{\text {eq }} / \tau_{\text {fo }}\right) \simeq 9.0 \times 10^{11}$. The present helicity fraction is then given by

$$
\epsilon_{0}= \begin{cases}1 & \text { for } \epsilon_{\mathrm{fo}}>\left(\frac{\tau_{\mathrm{rec}}}{\tau_{\mathrm{sc}}}\right)^{-\frac{2 \beta}{\beta+3}}, \\ \left(\frac{\tau_{\mathrm{rec}}}{\tau_{\mathrm{sc}}}\right)^{\frac{2 \beta}{\beta+3}} \epsilon_{\mathrm{fo}} & \text { for } \epsilon_{\mathrm{fo}}<\left(\frac{\tau_{\mathrm{rec}}}{\tau_{\mathrm{sc}}}\right)^{-\frac{2 \beta}{\beta+3}} \text { and } \tau_{\mathrm{rec}}>\tau_{\mathrm{sc}}, \\ \epsilon_{\mathrm{fo}} & \text { for } \tau_{\mathrm{rec}}<\tau_{\mathrm{sc}} .\end{cases}
$$

We shall also use $B_{0} \equiv \sqrt{2 \mathcal{E}_{0}}$ as the typical magnetic field strength today.

Now we can present predictions on the magnetic field properties today and compare them with the baryogenesis from the hypermagnetic helicity decay. From Eqs. (2.19), (3.6), (3.9), (3.10), and (3.11), we find that the properties of the present magnetic field, $\xi_{0}, B_{0}$, and $\epsilon_{0}$ determine the baryon asymmetry produced by the hypermagnetic helicity decay regardless of its evolution history as

$$
\bar{\eta}_{B} \simeq 10^{-10} \epsilon_{0}\left(\frac{\mathcal{C}}{10^{33} \mathrm{Mpc}^{-1} \mathrm{G}^{-2}}\right)\left(\frac{\xi_{0}}{10^{-9} \mathrm{Mpc}}\right)\left(\frac{B_{0}}{10^{-17} \mathrm{G}}\right)^{2} .
$$


The fiducial value, $\mathcal{C} \sim 10^{33} \mathrm{Mpc}^{-1} \mathrm{G}^{-2}$, is taken from Eq. (2.20). The constraints from the baryon isocurvature perturbation in terms of the present magnetic field properties can be derived in a similar way, but we need to specify the shape of the magnetic field spectrum to evaluate concretely. We perform the investigation in the next section.

Before closing this section, let us summarize the constraints on the cosmological magnetic fields other than those from the inhomogeneous BBN. First, once the system enters the cascade regime, the relation between the present magnetic field strength and coherence length is determined by their values at the recombination as

$$
\xi_{0}=\xi_{\mathrm{c}, \mathrm{ed}, \mathrm{rec}}=\left.a^{-1}\left(\frac{2 \mathcal{E}_{\mathrm{c}}}{\rho_{c}+p_{c}}\right)^{\frac{1}{2}}\right|_{T=T_{\mathrm{rec}}} t_{\mathrm{rec}} \simeq 0.6 \mathrm{pc}\left(\frac{B_{0}}{10^{-14} \mathrm{G}}\right) .
$$

and hence the primordial magnetic fields generated before the recombination should have the property, $\xi_{0} \gtrsim 0.6 \mathrm{pc}\left(B_{0} / 10^{-14} \mathrm{G}\right)$. As we have mentioned, since the magnetic fields contribute to additional relativistic energy density of the Universe, the BBN gives the upper bound on the comoving magnetic field strength when it occurs, $B_{\mathrm{c}, \mathrm{BBN}}<10^{-6} \mathrm{G}$ [73], regardless of the coherence length. Moreover, the CMB anisotropy imposes another upper bound on the cosmological magnetic field strength whose comoving coherence length is longer than Mpc scales as $B_{0}<10^{-9} \mathrm{G}$ [79-88] (summarized in Ref. [74]), since they would give additional contributions to the CMB anisotropy.

On the other hand, recent gamma-ray observations of blazars suggest the existence of the intergalactic magnetic fields [36-44]. For example, the Fermi collaboration reported the deficit of the secondary $\mathrm{GeV}$ cascade photons that should accompany the $\mathrm{TeV}$ photons from the blazars, which implies the existence of the intergalactic magnetic fields. The latest analysis suggests that the observations can be explained if the magnetic fields are strong enough [44],

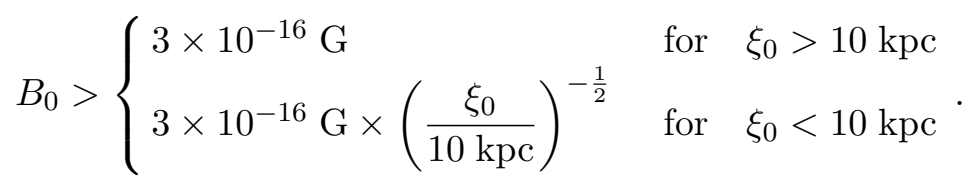

Note that Ref. [44] gives the constraint only for the coherence length $10^{2} \mathrm{pc}<\xi_{0}<10^{2} \mathrm{Mpc}$, and hence strictly speaking we can use the constraint only in these ranges, but we will use the extrapolated values as the reference. Comparing Eq. (3.14) to Eq. (3.12), the blazar observation can be explained by the primordial magnetic fields responsible for the present BAU only for $\epsilon_{0} \lesssim 10^{-9}$. In particular, if the magnetic fields have entered in the cascade regime before the recombination, from Eqs. (3.12) and (3.13) we obtain the condition for the BAU to be explained by the hypermagnetic helicity decay in terms of the present magnetic field properties as

$$
B_{0} \simeq 1 \times 10^{-17} \mathrm{G} \times \epsilon_{0}^{-\frac{1}{3}}, \quad \xi_{0} \simeq 6 \times 10^{-10} \mathrm{Mpc} \times \epsilon_{0}^{-\frac{1}{3}},
$$

regardless of the shape of the spectrum.

\section{Specific models}

In the previous sections, we have presented a formalism to explain the BAU from the hypermagnetic helicity decay in connection with the properties of the magnetic field both at the EWSB and the present. It turned out that the scenario is consistent with the IGMFs 
suggested by the blazar observations only when the helicity fraction is very small, $\epsilon \lesssim 10^{-9}$. From baryon isocurvature perturbations, we can already constrain the magnetic field coherence length roughly as $\xi_{\text {c,fo }} \lesssim k_{\mathrm{d}}^{-1}$ to avoid the deuterium overproduction. However, to apply the more concrete constraint described in $\S 3.1$, we need to specify the magnetic field spectra at the EWSB. In this section, we specify specific forms of the power spectra, which enable us to extract the general feature of the constraint. We also present appropriate constraints in terms of the present magnetic field properties.

\subsection{Delta function}

Let us first investigate the simplest choice of $S(k)$ and $A(k)=\epsilon S(k)$ at the EWSB, a monochromatic form described by the delta function.

$$
S(k)=\pi^{2} \frac{B_{\mathrm{c}, \mathrm{fo}}^{2}}{k_{\sigma}^{4}} \delta\left(k-k_{\sigma}\right), \quad A(k)=\epsilon_{\mathrm{fo}} S(k),
$$

so that the characteristic properties of the magnetic fields, as well as the net baryon asymmetry are given by

$$
\mathcal{E}_{\mathrm{c}, \mathrm{fo}}=\frac{1}{2} B_{\mathrm{c}, \mathrm{fo}}^{2}, \quad \xi_{\mathrm{c}, \mathrm{fo}}=k_{\sigma}^{-1}, \quad h_{\mathrm{c}, \mathrm{fo}}=\epsilon_{\mathrm{fo}} \xi_{\mathrm{c}, \mathrm{fo}} B_{\mathrm{c}, \mathrm{fo}}^{2}, \quad \bar{\eta}_{B}=\epsilon_{\mathrm{fo}} \mathcal{C} \xi_{\mathrm{c}, \mathrm{fo}} B_{\mathrm{c}, \mathrm{fo}}^{2}
$$

respectively. Note that the previous studies on the homogeneous part of the baryon asymmetry from the hypermagnetic helicity decay $[9,11]$ implicitly assumes such spectra.

The baryon isocurvature perturbation at the BBN is evaluated from Eq. (3.5) as

$$
\overline{S_{B, \mathrm{BBN}}^{2}}=\frac{1}{4}\left(\frac{1}{\epsilon_{\mathrm{fo}}^{2}}+1\right) D \xi_{\mathrm{c}, \mathrm{fo}}^{2}\left[2-2 D \xi_{\mathrm{c}, \mathrm{fo}}^{2}+D^{2} \xi_{\mathrm{c}, \mathrm{fo}}^{4}\left(1-e^{-\frac{2}{D \xi_{\mathrm{c}, \mathrm{fo}}}}\right)\right] .
$$

In the limit of large coherence length, $D \xi_{\mathrm{c}, \text { fo }_{\mathrm{o}}}^{2} \gg 1$,

$$
\overline{S_{B, \mathrm{BBN}}^{2}}=\frac{1}{3}\left(\frac{1}{\epsilon_{\mathrm{fo}}^{2}}+1\right)+\mathcal{O}\left(\left(D \xi_{\mathrm{c}, \mathrm{fo}}^{2}\right)^{-1}\right) .
$$

In this limit, the effect of neutron diffusion is ineffective and the condition (3.1) is never satisfied. In the opposite limit, $D \xi_{\text {c,fo }}^{2} \ll 1$, corresponding to small-scale magnetic fields, which is the case we are interested in so as to maintain the condition (3.1),

$$
\overline{S_{B, \mathrm{BBN}}^{2}}=\frac{1}{2}\left(\frac{1}{\epsilon_{\mathrm{fo}}^{2}}+1\right) D \xi_{\mathrm{c}, \mathrm{fo}}^{2}+\mathcal{O}\left(\left(D \xi_{\mathrm{c}, \mathrm{fo}}^{2}\right)^{2}\right) .
$$

A factor $\xi_{\text {c.fo }}^{2}$ appears in the leading term, convincing us that the shorter the coherence length of the fields is, the more suppressed the baryon isocurvature perturbations. This is because neutrons are more likely to smear out the inhomogeneity of baryon distribution. Needless to say, the delta-function type power spectrum in the wavenumber space does not mean a vanishing correlation on scales larger than the corresponding scale in the position space, and hence we acquire a suppressed but finite correlation even after smearing out by the neutron diffusion.

The condition for sufficiently small baryon isocurvature perturbation (Eq. (3.1)), with the condition that the present BAU is explained by the hypermagnetic helicity decay (Eq. 
(2.21)) being satisfied, gives the conditions in terms of the magnetic field properties at the EWSB as well as the present.

First we express the constraint in terms of the magnetic field properties at the EWSB. The condition for the MHD erasure of the small-scale magnetic fields (Eq. (3.6)) is written in terms of the physical magnetic field properties as

$$
B_{\mathrm{p}, \text { fo }} \lesssim 6 \times 10^{-3} \mathrm{GeV}^{2} \times\left(\frac{\xi_{\mathrm{p}, \text { fo }}}{10^{6} \mathrm{GeV}^{-1}}\right),
$$

which does not depend on the helicity fraction $\epsilon_{\mathrm{fo}}$. Here we have used the number of charged relativistic degrees of freedom $g_{* \text {,fo }}^{\text {ch }}=82.75$.

The condition for the magnetic field to generate the present BAU, Eq. (2.21), yields the lower bound of the magnetic field strength together with the requirement $\epsilon_{\mathrm{fo}} \leq 1$, as

$$
B_{\mathrm{p}, \text { fo }} \gtrsim 5 \times 10^{-3} \mathrm{GeV}^{2}\left(\frac{\xi_{\mathrm{p}, \mathrm{fo}}}{10^{6} \mathrm{GeV}^{-1}}\right)^{-\frac{1}{2}} .
$$

Conversely, the condition (2.21) allow us to determine the helicity fraction $\epsilon_{\mathrm{fo}}$ for a given magnetic field strength and coherence length. This leads to the lower bound of the magnetic field strength from the baryon isocurvature constraint (Eq. (3.1)) with Eq. (4.5) as

$$
B_{\mathrm{p}} \lesssim 3 \times 10^{-3} \mathrm{GeV}^{2}\left(\frac{\xi_{\mathrm{p}, \mathrm{fo}}}{10^{10} \mathrm{GeV}^{-1}}\right)^{-1}
$$

for $\epsilon_{\mathrm{fo}} \ll 1$, where we have used the fact that the neutron diffusion scale at the BBN corresponds to the proper wavenumber $k_{\mathrm{d} \text {,fo }}^{\mathrm{p}}=4.4 \times 10^{-15} \mathrm{GeV}$ at the EWSB.

The parameter space constrained by Eqs. (4.6), (4.7), and (4.8) (with the behavior at $\epsilon_{\mathrm{fo}} \sim 1$ being appropriately taken into account) is shown as the green shaded region of the right panel of Fig. 1. Here the smallest helicity fraction that satisfies all the conditions is found to be $\epsilon_{\mathrm{fo}} \simeq 2 \times 10^{-6}$.

Next, let us turn to the constraints expressed by the present IGMF properties. First of all, large-scale magnetic fields whose coherence length is larger than the one for the MHD turbulence (Eq. (3.13)) are ruled out. We have seen that such magnetic fields have evolved adiabatically with the comoving coherence length being unchanged. Then, it can be easily checked that it is impossible to satisfy $\xi_{\mathrm{c}, \text { fo }}<k_{\mathrm{d}}^{-1}$, which is required by the baryon isocurvature constraints (Eq. (3.1)), simultaneously satisfying the condition for the generation of the present BAU (Eq. (3.12)). For the IGMF whose properties lie in the line Eq. (3.13), there remains a possibility to satisfy the baryon isocurvature constraint since it has experieced the cascade process and the coherence length is much shorter at the EWSB. Since the relationship between the coherence length at the EWSB and that at the present depends on the value of $\beta$, the baryon isocurvature constraint today also depends on $\beta$. If $\beta>1$, the magnetic fields that satisfy the constraint at the EWSB (green shaded region in Fig. 1) become maximally helical until the recombination. This can be seen that in the $B-\xi$ figure, the magnetic field evolves with $B_{\mathrm{c}} \propto \xi_{\mathrm{c}}^{-(\beta+1) / 2}$ whereas the baryon isocurvature constraint yields $B_{\mathrm{c}} \propto \xi_{\mathrm{c}}^{-1}$ (Eq. (4.8)). In this case, the IGMF property is uniquely determined as

$$
B_{0} \simeq 1 \times 10^{-17} \mathrm{G}, \quad \xi_{0} \simeq 6 \times 10^{-4} \mathrm{pc} .
$$

The longest coherence length is obtained for the extreme choice, $\beta=0$, (which would be hard to realize, though), where the cascade is most effective. In such a case, the helicity fraction 
does not change so that the smallest one is $\epsilon_{0} \sim \epsilon_{\mathrm{fo}} \sim 2 \times 10^{-6}$. This uniquely determines the upper bound of the magnetic field strength and coherence length for $0 \leq \beta \leq 1$ as

$1 \times 10^{-17} \mathrm{G} \lesssim B_{0} \lesssim 7 \times 10^{-16} \mathrm{G} \times\left(\frac{\tau_{\text {rec }}}{\tau_{\text {fo }}}\right)^{-\frac{\beta+1}{\beta+3}+\frac{1}{3}}, 6 \times 10^{-4} \mathrm{pc} \lesssim \xi_{0} \lesssim 0.07 \mathrm{pc} \times\left(\frac{\tau_{\text {rec }}}{\tau_{\text {fo }}}\right)^{\frac{2}{\beta+3}-\frac{2}{3}}$,

with $\tau_{\text {rec }} / \tau_{\text {fo }} \simeq 9 \times 10^{11}$ where the lower bound is for the case $\epsilon_{0}=1$. The allowed region is depicted as the green line in the right panel of Fig. 1. The constraint (4.10) falls below the blazar constraint (Eq. (3.14)), $B_{0} \lesssim 3 \times 10^{-14} \mathrm{G}$ on the line of the MHD turbulence (Eq. (3.13)), by a factor of $\mathcal{O}\left(10^{2}\right)$.
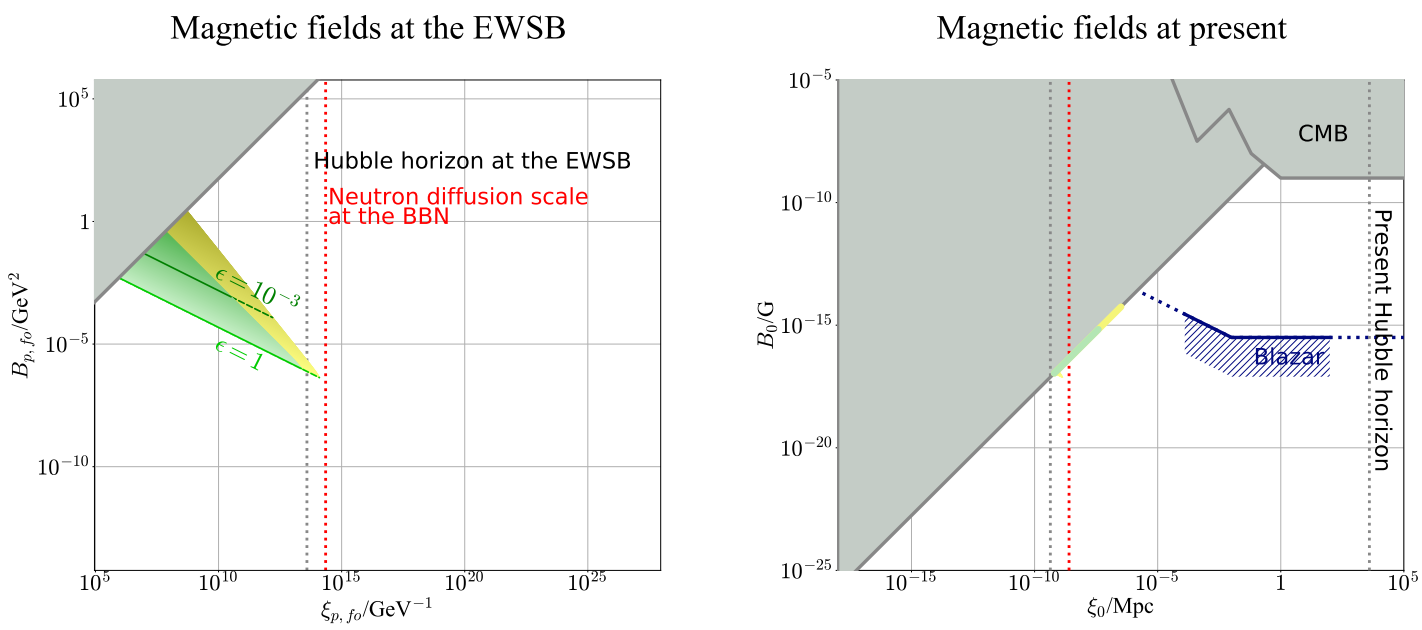

Figure 1. Left. Constraints on the magnetic fields at the EWSB, Equations. (4.6), (4.7), (4.8), and (4.18) are shown in terms of the physical quantities. Right. Constraints on the present magnetic fields, Eqs. (3.15), (4.10), and (4.20) are shown. The navy thick line, with its lower side hatched, is the lower bounds suggested by blazar observations [44]. The navy dotted lines are an extrapolation of Ref. [44]. In both panels, the gray shaded regions above the eddy turnover scales are the parameter space inconsistent with the MHD evolution. The green shaded region and line are the allowed parameter regions for the delta-function model, while the yellow shaded region and line are the one for the power-law model with $\alpha=0$.

\subsection{A Power-law spectrum with an exponential cutoff}

Next we study a more realistic form of the spectrum, a power-law spectrum with an exponential cutoff,

$$
S(k)=\frac{2 \pi^{2}}{\Gamma\left(\frac{5+\alpha}{2}\right)} \frac{B_{\mathrm{c}, \mathrm{fo}}^{2}}{k_{\sigma}^{5}}\left(\frac{k}{k_{\sigma}}\right)^{\alpha} \exp \left[-\left(\frac{k}{k_{\sigma}}\right)^{2}\right], \quad A(k)=\epsilon_{\mathrm{fo}} S(k) .
$$

In this case, characteristic properties of the magnetic fields, as well as the net baryon asymmetry produced by the fields, are given by

$$
\mathcal{E}_{\mathrm{c}, \mathrm{fo}}=\frac{1}{2} B_{\mathrm{c}, \mathrm{fo}}^{2}, \quad \xi_{\mathrm{c}, \mathrm{fo}}=\left(\zeta_{\alpha} k_{\sigma}\right)^{-1}, \quad h_{\mathrm{c}, \mathrm{fo}}=\epsilon_{\mathrm{fo}} \xi_{\mathrm{c}, \mathrm{fo}} \mathcal{E}_{\mathrm{c}, \mathrm{fo}}, \quad \bar{\eta}_{B}=\epsilon_{\mathrm{fo}} \mathcal{C} \xi_{\mathrm{c}, \mathrm{fo}} B_{\mathrm{c}, \mathrm{fo}}^{2}
$$


respectively, where $\zeta_{\alpha} \equiv \frac{\Gamma\left(\frac{5+\alpha}{2}\right)}{\Gamma\left(2+\frac{\alpha}{2}\right)}$. Here we restrict ourselves as $\alpha>-4$ otherwise the energy density and coherence length are subject to infrared divergences. Such a spectrum is introduced, for example, in Ref. [6, 7], and we expect that this single-power law with exponential decay model captures the essential properties of a broader class of spectrum including the one [89] that has a Batchelor spectrum at small $k$, a Kolmogorov spectrum at intermediate $k$, and an exponential cutoff at large $k$ because the baryon isocurvature constraints are mainly determined by the peak and the infrared tail of the spectrum. Batchelor spectrum cut off at large wavenumber corresponds to $\alpha=0$ in our notation, so we are mainly interested in the parameter range around $\alpha \sim 0$.

In this case, the baryon isocurvature perturbation at the BBN is given from Eq. (3.5) as

$$
\begin{aligned}
\overline{S_{B, \mathrm{BBN}}^{2}}=\frac{\left(\zeta_{\alpha} \xi_{\mathrm{c}, \mathrm{fo}}\right)^{2} D}{2 \Gamma\left(2+\frac{\alpha}{2}\right)^{2}} \int d q_{1} d q_{2} q_{1}^{\alpha} q_{2}^{\alpha} e^{-q_{1}^{2}-q_{2}^{2}} \\
\sum_{ \pm}\left(e ^ { - \frac { ( q _ { 1 } \pm q _ { 2 } ) ^ { 2 } } { 2 ( \zeta _ { \alpha } \xi _ { \mathrm { c } , \mathrm { fo } } ) ^ { 2 } D } } \left\{\left(1 \mp \frac{1}{\epsilon_{\mathrm{fo}}^{2}}\right)\left(q_{1} \mp q_{2}\right)^{2}\left[q_{1} q_{2} \pm\left(\zeta_{\alpha} \xi_{\mathrm{c}, \mathrm{fo}}\right)^{2} D\right]\right.\right. \\
\left.\left.\mp\left(2+\frac{1}{\epsilon_{\mathrm{fo}}^{2}} \frac{q_{1}^{2}+q_{2}^{2}}{q_{1} q_{2}}\right)\left[\left(\zeta_{\alpha} \xi_{\mathrm{c}, \mathrm{fo}}\right)^{2} D\right]^{2}\right\}\right)
\end{aligned}
$$

where we have introduced a dimensionless wavenumber $q_{i}=\zeta_{\alpha} \xi_{\mathrm{c}, \mathrm{fo}} k_{i}$. In the limit of large coherence length, for $\alpha>-3,\left(\zeta_{\alpha} \xi_{\mathrm{c}, \mathrm{fo}}\right)^{2} D \gg 1$,

$$
\overline{S_{B, \mathrm{BBN}}^{2}}=\frac{1}{3}\left[\frac{\Gamma\left(\frac{3+\alpha}{2}\right) \Gamma\left(\frac{5+\alpha}{2}\right)}{\Gamma\left(2+\frac{\alpha}{2}\right)^{2} \epsilon_{\mathrm{fo}}^{2}}+1\right]+\mathcal{O}\left(\left[\left(\zeta_{\alpha} \xi_{\mathrm{c}, \mathrm{fo}}\right)^{2} D\right]^{-1}\right),
$$

where the terms with positive powers of $\left(\zeta_{\alpha} \xi_{\text {c,fo }}\right)^{2} D$ are canceled out by the exponential factor. In the case $\alpha \leq-3$, since the integrand behaves as $k_{1}^{\alpha+2} k_{2}^{\alpha+2} \times \mathcal{O}\left(k_{1}^{2}, k_{2}^{2}\right) /\left(\epsilon_{\mathrm{fo}}^{2}\left(\zeta_{\alpha} \xi_{\mathrm{c}, \mathrm{fo}}\right)^{2} D\right)$, the volume average of the baryon isocurvature perturbation diverges at the infrared as

$$
\overline{S_{B, \mathrm{BBN}}^{2}} \sim \frac{1}{\epsilon_{\mathrm{fo}}^{2}}\left(\zeta_{\alpha} \xi_{\mathrm{c}, \mathrm{fo}} \Lambda_{\mathrm{IR}}\right)^{3+\alpha},
$$

where $\Lambda_{\mathrm{IR}}$ is the infrared cutoff, and hence it becomes unacceptably large for $\left(\zeta_{\alpha} \xi_{\mathrm{c}, \mathrm{fo}}\right)^{2} D \gg 1$. In this limit, once more, the effect of neutron diffusion is ineffective and the condition (3.1) is never satisfied.

In the other limit $\left(\zeta_{\alpha} \xi_{\text {c,fo }}\right)^{2} D \ll 1$, which is the case when the condition (3.1) can be satisfied, by using the saddle point approximation, we can approximate the volume average of the baryon isocurvature perturbation for $\alpha>-5 / 2$ as

$$
\overline{S_{B, \mathrm{BBN}}^{2}}=\frac{\sqrt{\pi} 2^{-2-\alpha} \Gamma\left(\frac{5}{2}+\alpha\right)}{\Gamma\left(2+\frac{\alpha}{2}\right)^{2}}\left(\frac{1}{\epsilon_{\mathrm{fo}}^{2}}+1\right)\left[\left(\zeta_{\alpha} \xi_{\mathrm{c}, \mathrm{fo}}\right)^{2} D\right]^{\frac{3}{2}}+\mathcal{O}\left(\left[\left(\zeta_{\alpha} \xi_{\mathrm{c}, \mathrm{fo}}\right)^{2} D\right]^{\frac{5}{2}}\right) .
$$

A factor $\xi_{\mathrm{c}, \mathrm{fo}}^{-3}$ appears in the leading term, meaning that the neutron dissipation effect is more effective than that in delta-function model, in which case the corresponding factor is $k_{\sigma}^{-2}$. See Appx. B for more discussion on the interpretation how these difference appears.

The apparent divergence for $-3<\alpha \leq-5 / 2$ is due to the breakdown of the validity of the saddle point approximation. Since the IR behavior of the integrand is the same as that 
seen in the case with $\left(\zeta_{\alpha} \xi_{\mathrm{c}, \text { fo }}\right)^{2} D \gg 1$, with $\left(\zeta_{\alpha} \xi_{\mathrm{c}, \text { fo }}\right)^{-2} D^{-1}$ being taken as the "UV" cutoff, we can estimate as

$$
\overline{S_{B, \mathrm{BBN}}^{2}} \sim \frac{1}{\epsilon_{\mathrm{fo}}^{2}}\left[\left(\zeta_{\alpha} \xi_{\mathrm{c}, \mathrm{fo}}\right)^{2} D\right]^{\frac{3+\alpha}{2}} .
$$

The divergence at $\alpha<-3$ is the physical infrared divergence and the volume average of the baryon isocurvature perturbation behaves as Eq. (4.15) in the same way to the case with $\left(\zeta_{\alpha} \xi_{\text {c,fo }}\right)^{2} D \gg 1$. Hereafter we will focus on the case with $\alpha>-5 / 2$ for simplicity .

Now we are ready to present the constraints on the properties of the magnetic field both at the EWSB as well as those at the present. As for the magnetic fields at the EWSB, the constraints derived from the MHD, (4.6), and from baryon-number generation, (4.7), apply in the same way as in the the delta-function spectrum. Hence the baryon isocurvature constraint can be derived in a similar way to the previous subsection. By combining Eqs. (2.21) and (4.16), the baryon isocurvature constraint Eq. (3.3) is rewritten in a form of the constraint on the magnetic field strength as

$$
B_{\mathrm{p}, \text { fo }} \lesssim 3 \times 10^{-2} \mathrm{GeV}^{2} \times 2^{\frac{\alpha}{4}} \times \frac{\Gamma\left(2+\frac{\alpha}{2}\right)^{\frac{5}{4}}}{\Gamma\left(\frac{5}{2}+\alpha\right)^{\frac{1}{4}} \Gamma\left(\frac{5+\alpha}{2}\right)^{\frac{3}{4}}} \times\left(\frac{\xi_{\mathrm{p}, \text { fo }}}{10^{10} \mathrm{GeV}^{-1}}\right)^{-\frac{5}{4}},
$$

for $\epsilon_{\text {fo }} \ll 1$. The parameter space for $\alpha=0$ constrained by Eqs. (4.6), (4.7), and (4.18) (with the behavior at $\epsilon_{\mathrm{fo}} \sim 1$ being appropriately taken into account) is shown in the yellow shaded region of the right panel of Fig. 1. The smallest helicity fraction that satisfies all the conditions is evaluated as

$$
\epsilon_{\mathrm{fo}}=\epsilon_{\mathrm{fo}}^{\min } \equiv 1 \times 10^{-8} \times 2^{-\frac{\alpha}{3}} \frac{\Gamma\left(\frac{5}{2}+\alpha\right)^{\frac{1}{3}} \Gamma\left(\frac{5+\alpha}{2}\right)}{\Gamma\left(2+\frac{\alpha}{2}\right)^{\frac{5}{3}}} .
$$

The constraints on the present magnetic field are also given in the same way as in the delta-function spectrum. We see that most part of the parameter region in which present magnetic fields have never entered the MHD turbulence does not satisfy the baryon isocurvature constraints and almost only those which lie in the line Eq. (3.13) have the possibility to satisfy them. From the $B-\xi$ figure (Fig. 1), we can see that the magnetic fields that satisfy the baryon isocurvature constraint at the EWSB becomes maximally helical if they evolve more rapidly than $B_{\mathrm{c}} \propto \xi_{\mathrm{c}}^{-5 / 4}$ (See also Eq. (4.18)). This corresponds to the cascade parameter $\beta>3 / 2$. In such a case, again, the IGMF property is uniquely determined as Eq. (4.9). The strongest IGMF is obtained for $\beta=0$ and $\epsilon_{\mathrm{fo}} \sim \epsilon_{0}=\epsilon_{\mathrm{fo}}^{\min }$, which gives the parameter spaces of the IGMF properties that can explain the BAU satisfying the baryon isocurvature constraint as

$1 \times 10^{-17} \mathrm{G} \lesssim B_{0} \lesssim 3 \times 10^{-15} \mathrm{G} \times\left(\frac{\tau_{\text {rec }}}{\tau_{\text {fo }}}\right)^{-\frac{\beta+1}{\beta+3}+\frac{1}{3}}, \quad 6 \times 10^{-4} \mathrm{pc} \lesssim \xi_{0} \lesssim 0.3 \mathrm{pc} \times\left(\frac{\tau_{\text {rec }}}{\tau_{\text {fo }}}\right)^{\frac{2}{\beta+3}-\frac{2}{3}}$,

with $\alpha=0$. Note that the constraints are quite insensitive to the value of $\alpha$. These parameter space is shown in the yellow line in the right panel of Fig. 1.

Figure 2 shows the $\epsilon_{0}$ dependence of the IGMF strength on the MHD line Eq. (3.13). Yellow and green lines represent the parameter region where the baryon isocurvature constraint is satisfied for $\beta=0$ in the case of the power-law spectrum with an exponential cutoff and the delta-function spectrum, respectively. For larger $\beta$, the maximum field strength becomes 


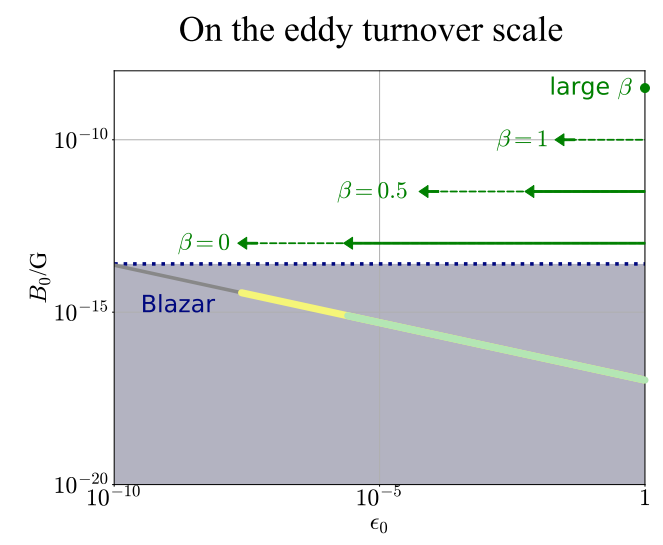

Figure 2. The constraints on the present magnetic fields on the eddy turnover scale, Eqs. (4.10) and (4.20), are shown as functions of the present helicity fraction. The thick green line is the allowed parameter region for the delta-function model, while the thick yellow line is allowed for the power-law model with $\alpha=0$, with the evolution parameter $\beta=0$. The navy dotted line shows the lower limit of the field strength extrapolated from the blazar observation [44]. The green arrows show the edges of the allowed regions for each $\beta$. For $\beta \gtrsim 1$, only around $B_{0}=1 \times 10^{-17} \mathrm{G}$ with $\epsilon_{0}=1$ is allowed in both models.

smaller. In particular, for $\beta>1$, which would be often realized according to Ref. [77], all the primordial magnetic fields that generate the correct BAU while satisfying the baryon isocurvature constraint reaches at $B_{0} \simeq 1 \times 10^{-17} \mathrm{G}$. Comparing to the gray shaded region, where the IGMF cannot explain the blazar observation, we conclude that the primordial magnetic fields that generate the correct BAU alone cannot explain the blazar observations regardless of its initial helicity or the shape of the spectrum. Note that the spectrum of baryon isocurvature perturbations has more power on the smaller wavenumber modes than that of the energy density, and hence it is less sensitive to the behavior of the ultraviolet decay. Therefore, all the existing magnetogenesis mechanisms that explain the present BAU, such as the axion inflation [26-31], the chiral plasma instability [32], or the Affleck-Dine mechanism [33] are subject to this constraint, since each of these models produces helical hypermagnetic fields at its own characteristic scale and they evolve according to MHD so that their infrared spectrum is likely to become that of Batchelor spectrum. One may wonder if it might be possible for some inflation models to generate partially helical magnetic fields at Mpc scales today while generating the net BAU, but such a possibility is now ruled out because of the excessive baryon isocurvature perturbation.

\section{Baryon isocurvature constraints on non-helical hypermagnetic fields}

Thus far we have discussed the baryon isocurvature constraint on the cosmological magnetic fields in the case they are responsible for the net BAU. Since not only the helical part but also the non-helical part sources the baryon isocurvature perturbation, we can universally apply what we discussed in the previous sections to non-helical hypermagnetic fields supposing the net BAU is generated by other mechanism, as long as magnetic fields existed before the EWSB. In this section, we give the constraints on the non-helical hypermagnetic fields $\epsilon_{\mathrm{fo}}=\epsilon_{0}=0$ from the baryon isocurvature perturbation. 
The volume average of the baryon isocurvature pertuerbation in this case can be evaluated by setting $\epsilon_{\mathrm{fo}}=0$ and using the observed value of $\bar{\eta}_{B}$ in Eq. (3.5) as

$$
\overline{S_{B, \mathrm{BBN}}^{2}}= \begin{cases}\frac{\mathcal{C}^{2} \xi_{\mathrm{c}, \mathrm{fo}}^{4} B_{\mathrm{c}, \mathrm{fo}}^{4}}{2 \bar{\eta}_{B}^{2}} D+\mathcal{O}\left(\left(\xi_{\mathrm{c}, \mathrm{fo}}^{2} D\right)^{2}\right)(<0.016), & \text { for } \quad \xi_{\mathrm{c}, \mathrm{fo}}^{2} D \ll 1, \\ \frac{\mathcal{C}^{2} \xi_{\mathrm{c}, \mathrm{fo}}^{2} B_{\mathrm{c}, \mathrm{fo}}^{4}}{3 \bar{\eta}_{B}^{2}}+\mathcal{O}\left(\left(\xi_{\mathrm{c}, \mathrm{fo}}^{2} D\right)^{-1}\right)(<0.016), & \text { for } \quad \xi_{\mathrm{c}, \mathrm{fo}}^{2} D \gg 1\end{cases}
$$

for the delta-function power spectrum and

$$
\overline{S_{B, \mathrm{BBN}}^{2}}=\left\{\begin{array}{c}
\frac{\sqrt{\pi} 2^{-\alpha} \Gamma\left(\frac{5}{2}+\alpha\right) \mathcal{C}^{2} \xi_{\mathrm{c}, \mathrm{fo}}^{5} B_{\mathrm{c}, \mathrm{fo}}^{4}}{4 \Gamma\left(2+\frac{\alpha}{2}\right)^{2} \bar{\eta}_{B}^{2}}\left(\zeta_{\alpha}^{2} D\right)^{\frac{3}{2}}+\mathcal{O}\left(\left[\left(\zeta_{\alpha} \xi_{\mathrm{c}, \mathrm{fo}}\right)^{2} D\right]^{\frac{5}{2}}\right)(<0.016), \\
\text { for } \quad\left(\zeta_{\alpha} \xi_{\mathrm{c}, \mathrm{fo}}\right)^{2} D \ll 1 \\
\frac{\Gamma\left(\frac{3+\alpha}{2}\right) \Gamma\left(\frac{5+\alpha}{2}\right) \mathcal{C}^{2} \xi_{\mathrm{c}, \mathrm{fo}}^{2} B_{\mathrm{c}, \mathrm{fo}}^{4}}{3 \Gamma\left(2+\frac{\alpha}{2}\right) \bar{\eta}_{B}^{2}}+\mathcal{O}\left(\left[\left(\zeta_{\alpha} \xi_{\mathrm{c}, \mathrm{fo}}\right)^{2} D\right]^{-1}\right)(<0.016), \\
\text { for } \quad\left(\zeta_{\alpha} \xi_{\mathrm{c}, \mathrm{fo}}\right)^{2} D \gg 1
\end{array}\right.
$$

for the power law spectrum with an exponential cutoff. In terms of physical quantities at the EWSB, the magnetic field strength are then constrained as

$$
B_{\mathrm{p}, \mathrm{fo}} \lesssim\left\{\begin{array}{l}
1 \times 10^{-7} \mathrm{GeV}^{2}\left(\frac{\xi_{\mathrm{p}, \mathrm{fo}}}{2 \times 10^{14} \mathrm{GeV}^{-1}}\right)^{-1}, \quad \text { for } \quad \xi_{\mathrm{p}, \mathrm{fo}} \ll \sqrt{\frac{2}{3}} k_{\mathrm{d}, \mathrm{fo}}^{\mathrm{p}-1}, \\
2 \times 10^{-7} \mathrm{GeV}^{2}\left(\frac{\xi_{\mathrm{p}, \mathrm{fo}}}{2 \times 10^{14} \mathrm{GeV}^{-1}}\right)^{-\frac{1}{2}}, \quad \text { for } \xi_{\mathrm{p}, \mathrm{fo}} \gg \sqrt{\frac{2}{3}} k_{\mathrm{d}, \mathrm{fo}}^{\mathrm{p}-1}
\end{array}\right.
$$

for the delta-function model, where $\sqrt{\frac{2}{3}} k_{\mathrm{d}, \mathrm{fo}}^{\mathrm{p}}{ }^{-1} \simeq 2 \times 10^{14} \mathrm{GeV}^{-1}$. The constraints for the power-law model, Eqs. (5.2), read

$$
B_{\mathrm{p}, \text { fo }} \lesssim\left\{\begin{array}{l}
1 \times 10^{-7} \mathrm{GeV}^{2}\left(\frac{\xi_{\mathrm{p}, \mathrm{fo}}}{2 \times 10^{14} \mathrm{GeV}^{-1}}\right)^{-\frac{5}{4}} \gamma_{1 \alpha}^{\frac{1}{4}}, \text { for } \xi_{\mathrm{p}, \text { fo }} \ll \sqrt{\frac{2}{3}}\left(\zeta_{\alpha} k_{\mathrm{d}, \mathrm{fo}}^{\mathrm{p}}\right)^{-1}, \\
2 \times 10^{-7} \mathrm{GeV}^{2}\left(\frac{\xi_{\mathrm{p}, \mathrm{fo}}}{2 \times 10^{14} \mathrm{GeV}^{-1}}\right)^{-\frac{1}{2}} \gamma_{2 \alpha}^{\frac{1}{4}}, \text { for } \xi_{\mathrm{p}, \text { fo }} \gg \sqrt{\frac{2}{3}}\left(\zeta_{\alpha} k_{\mathrm{d}, \mathrm{fo}}^{\mathrm{p}}\right)^{-1},
\end{array}\right.
$$

respectively, where we have defined $\gamma_{1 \alpha} \equiv \frac{2^{\alpha} \Gamma\left(2+\frac{\alpha}{2}\right)^{5}}{\Gamma\left(\frac{5+\alpha}{2}\right)^{3} \Gamma\left(\frac{5}{2}+\alpha\right)}$ and $\gamma_{2 \alpha} \equiv \frac{\Gamma\left(2+\frac{\alpha}{2}\right)}{\Gamma\left(\frac{3+\alpha}{2}\right) \Gamma\left(\frac{5+\alpha}{2}\right)}$.

The parameter space satisfying these constraints is shown in the left panel of Fig. 3 in green and yellow for the delta-function model and the power-law model with an exponential cutoff, respectively. Combining with the MHD turbulence condition at the EWSB (Eq. (4.6)), we find the upper bound of the magnetic field strength at the EWSB as

$$
B_{\mathrm{p}, \mathrm{fo}}^{\max } \simeq \begin{cases}0.4 \mathrm{GeV}^{2}, & \text { for delta function, } \\ 2 \mathrm{GeV}^{2} \times \gamma_{1 \alpha}^{-\frac{1}{9}}, & \text { for power law, }\end{cases}
$$


respectively. From the second lines of Eqs. (5.3) and (5.4), we can explicitly see that if the coherence length of the magnetic field at the EWSB is larger than the neutron diffusion scale, the effect of neutron diffusion is so small that the volume average of the inhomogeneity of baryon distribution at the $\mathrm{BBN}$ is determined only by $\xi_{\mathrm{c}, \mathrm{fo}} B_{\mathrm{c}, \mathrm{fo}}^{2}$ practically. This is because the amplitude of spatially-dependent component of the baryon asymmetry is still given by $\mathcal{C}|\boldsymbol{A}| \cdot|\boldsymbol{B}| \sim \mathcal{C} \xi_{\mathrm{c}, \mathrm{fo}_{\mathrm{o}}} B_{\mathrm{c}, \mathrm{fo}}^{2}$ without the factor of the helicity fraction $\epsilon_{\mathrm{fo}}$, as can be seen in Eq. (2.22) regardless of the shape of the power spectrum. On the other hand, in the case the coherence length of the magnetic fields is shorter than the neutron diffusion scale, the constraint depends on the shape of the spectrum. See Appendix B for analysis in the position space.

Translating these conditions to the present magnetic fields, we obtain the following constraints on the IGMF. Large-scale magnetic fields compared with the eddy turnover scale at the recombination, Eq. (3.13), evolve adiabatically from EWSB until today, and hence the constraints for the magnetic fields at the EWSB Eqs. (5.3) and (5.4) is directly translated as

$$
B_{0} \lesssim \begin{cases}3 \times 10^{-18} \mathrm{G}\left(\frac{\xi_{0}}{0.0025 \mathrm{pc}}\right)^{-\frac{1}{2}}, & \text { for delta function, } \\ 4 \times 10^{-18} \mathrm{G} \times \gamma_{2 \alpha}^{\frac{1}{4}}\left(\frac{\xi_{0}}{0.0025 \mathrm{pc}}\right)^{-\frac{1}{2}}, & \text { for power law. }\end{cases}
$$

Note that the neutron diffusion scale accidentally coincides the scale where the constraint line intersects with the line for the MHD turbulence scale.

On the other hand, the magnetic fields, which had a shorter coherence length originally, gradually start to evolve according to the cascade law and reach at the line that is determined by the eddy turnover scale at the recombination (Eq. (3.13)). With a similar consideration as in the previous section, we find that the strongest magnetic fields at the present is obtained in the case with the strongest magnetic fields at the EWSB (Eq. (5.5)) for the milder decay with smaller $\beta$ or in the case that magnetic fields enter the turbulence regime just before the recombination for the stronger decay with larger $\beta$. As a consequence, we obtain the upper bound of the magnetic field today as

$$
B_{0} \lesssim \begin{cases}\max \left[6 \times 10^{-16} \mathrm{G} \times\left(\frac{\tau_{\text {rec }}}{\tau_{\text {fo }}}\right)^{\frac{1}{3}-\frac{\beta+1}{\beta+3}}, 6 \times 10^{-18} \mathrm{G}\right], & \text { for delta function, } \\ \max \left[3 \times 10^{-15} \mathrm{G} \times \gamma_{1 \alpha}^{-\frac{1}{9}} \times\left(\frac{\tau_{\text {rec }}}{\tau_{\text {fo }}}\right)^{\frac{1}{3}-\frac{\beta+1}{\beta+3}}, 8 \times 10^{-18} \mathrm{G} \times \gamma_{2 \alpha}^{\frac{1}{6}}\right], & \text { for power law. }\end{cases}
$$

The right panel of Fig. 3 shows the parameter spaces that satisfy the baryon isocurvature constraint. Green and yellow shaded regions denote those for the delta-function model and power law with exponential cutoff model (for $\alpha=0$ ), respectively. The strongest magnetic fields are obtained at the MHD turbulence line (Eq. (3.13)), with the cascade law with $\beta=0$. Compared with the current observations, once more, the upper bounds are below the blazar constraint, $B_{0} \lesssim 3 \times 10^{-14} \mathrm{G}$, by a factor of $\mathcal{O}(10-100)$. On the contrary, the galaxy scales, $\xi_{0} \sim 0.1-10 \mathrm{kpc}$, the upper bound of the magnetic fields is roughly $B_{0} \lesssim 10^{-20} \mathrm{G}$. This would be sufficient to be the seed magnetic fields for the magnetic fields in the galaxy and galaxy cluster [90]. 

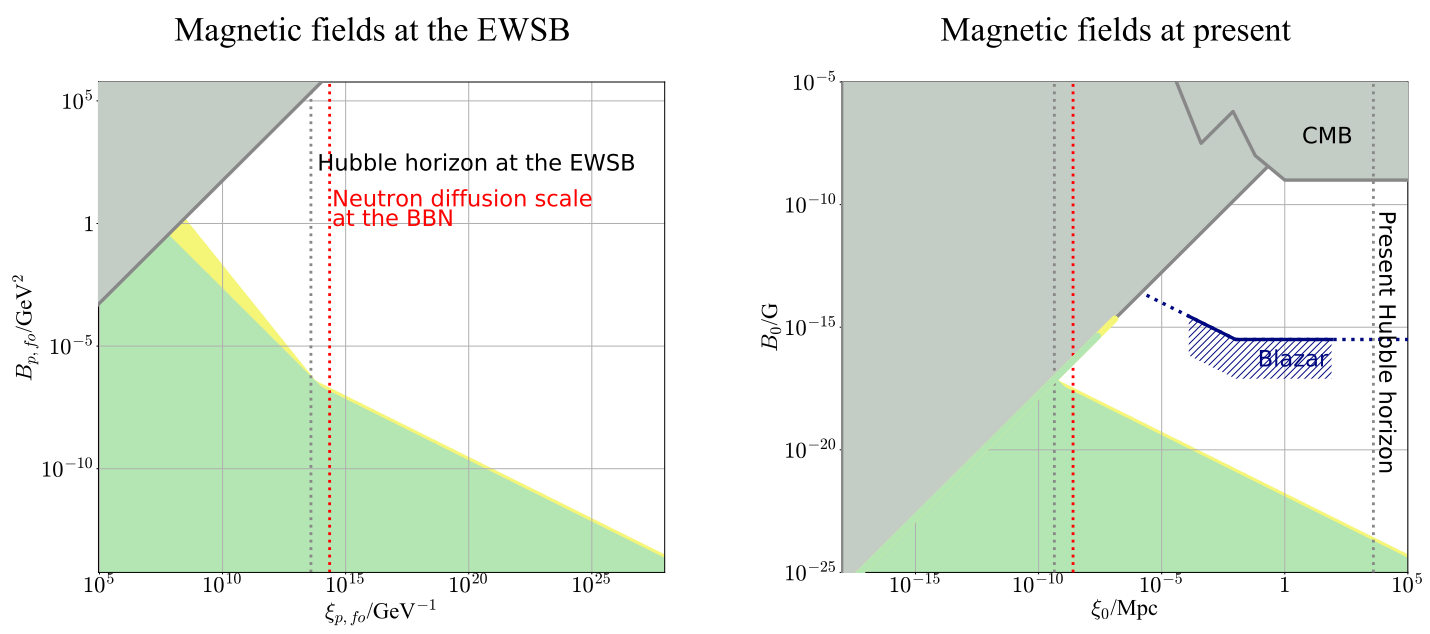

Figure 3. Constraints on the magnetic fields at the EWSB, (5.3) and (5.4) in terms of the physical quantities (Left) and those on the present magnetic fields, Eqs. (5.6) and (5.7) (Right) are shown. The navy line in the right panel is the lower bounds of the IGMFs suggested by the blazar observations [44] with an extrapolation (dotted line). In both panels, the gray shaded regions are the parameter spaces that are inconsistent with the MHD. The green shaded regions are the allowed parameter spaces for the delta-function model, while the yellow shaded regions are those for the power-law model with $\alpha=0$.

Figures 3 exhibit quite different allowed regions compared with Fig. 1 because the net baryon asymmetry is fixed independently of the properties of the magnetic fields. Since weaker magnetic fields that cannot generate the observed BAU is now allowed, viable parameter spaces are widely open. However, since the baryon isocurvature perturbation is mainly carried by the non-helical part of the hypermagnetic fields, the constraints do not change much for the stronger magnetic fields that can generate correct amount of the BAU, which lie on the line that is determined by the eddy turnover scale (Eq. (3.13)).

Thus far we have not discussed the inflationary magnetogenesis that may have generated a relatively flat spectrum $\alpha<-4$ up to the Mpc scales today with a comoving infrared cutoff $H_{0}<k_{\mathrm{IR}}<k_{\mathrm{d}}$ with $H_{0}$ being the present Hubble parameter. In this case, we can write the power spectrum as

$$
S(k)=\frac{\left(B_{\mathrm{c}, \mathrm{fo}_{\mathrm{o}}}^{\mathrm{IR}}\right)^{2}}{k_{\mathrm{IR}}^{5}}\left(\frac{k}{k_{\mathrm{IR}}}\right)^{\alpha} \exp \left[-\left(\frac{k}{k_{\sigma}}\right)^{2}\right] .
$$

with $\epsilon_{\mathrm{fo}}=0$. Here $B_{\mathrm{c}, \mathrm{fo}}^{\mathrm{IR}}$ represents the magnetic field strength at the infrared cutoff. In a similar way that is discussed in Eq. (4.15), we can evaluate the volume average of the baryon isocurvature perturbation as

$$
\overline{S_{B, \mathrm{BBN}}^{2}} \sim \frac{\mathcal{C}^{2}\left(B_{\mathrm{c}, \mathrm{fo}}^{\mathrm{IR}}\right)^{4}}{\bar{\eta}_{B}^{2} k_{\mathrm{IR}}^{2}},
$$

which is almost equal to those for the delta-function like model or the power law with exponential cutoff model. Therefore, we can conclude that even in this case, the magnetic field strength at the IR cutoff should be tiny so that the spectrum at the IR cut off comes below the constraint given in Eq. (5.6). We conclude that inflationary magnetogenesis with a relatively 
flat spectrum alone also cannot explain the blazar observations if they are generated before the EWSB unless there are an amplification process.

\section{Conclusion and Discussion}

Baryogenesis from the hypermagnetic helicity decay is an attractive scenario since it does not require any new physical degrees of freedom beyond the SM in the mechanism itself. If this mechanism is responsible for the present BAU, physics beyond the SM is required for the mechanism to produce the appropriate helical hypermagnetic fields.

In this paper, we have studied constraints on the mechanism from baryon isocurvature perturbations. Since the baryon fluctuations are produced in response to the coherence length of the hypermagnetic fields, large scale hypermagnetic fields are disfavored otherwise it might produce the anti-matter region, or at least, spoils the success of the standard BBN. One important essence is that non-helical part of the hypermagnetic fields contributes to the baryon isocurvature perturbation while only their helical part contributes to the homogeneous part of the net baryon asymmetry. Cosequently, baryon isocurvature perturbations just with $\mathcal{O}(1) \%$ level on scales larger than the comoving neutron diffusion scale at BBN is ruled out because the deuteriums are overproduced due to the second order effect.

Our main results are summarized in Fig. 1. We find that the magnetic fields whose coherence length at the EWSB is larger than the comoving neutron diffusion scale at BBN are completely ruled out since generation of the correct amount of the BAU and the small enough baryon isocurvature perturbation cannot be satisfied simultaneously. In summary, for the primordial magnetic field to produce the $\mathrm{BAU}$, the coherence length and the strength are constrained as $10^{6} \mathrm{GeV}^{-1}<\xi_{\mathrm{p}}<10^{15} \mathrm{GeV}^{-1}$ and $10^{-7} \mathrm{GeV}^{2}<B_{\mathrm{p}}<10 \mathrm{GeV}^{2}$ in physical quantity at the EWSB. Such magnetic fields evolve according to the MHD cascade processes and remain as the IGMFs. For the typical cascade parameter $\beta \simeq 2$, the present IGMF property is uniquely determined as $\xi_{0} \simeq 10^{-3} \mathrm{pc}$ and $B_{0} \simeq 10^{-17} \mathrm{G}$ with being maximally helical today, regardless of the shape of the magnetic field spectrum. Even allowing relatively unrealistic value of $\beta$ up to 0 , we find the present IGMF must satisfy $10^{-3} \mathrm{pc}<\xi_{0}<1 \mathrm{pc}$ and $10^{-17} \mathrm{G}<B_{0}<10^{-14} \mathrm{G}$, on the line Eq. (3.13), which is determined by the eddy turnover scale. It is found that the relationship among the parameters that describe the magnetic fields, that is, $\left.B_{0}, \xi_{0}\right)$, and $\epsilon_{0}$ is uniquely determined by the condition to generate the correct amount of BAU (Eq. (3.12)) and to satisfy the MHD evolution (Eq. (3.13)) as $B_{0} \propto \xi_{0} \propto \epsilon_{0}^{-\frac{1}{3}}$, leaving only one degree of freedom to the IGMFs regardless of their spectrum or the evolution history. While this would act as the important consistency relation to test this scenario, the upper bound from the baryon isocurvature perturbation is (at most) marginally below the present lower bound of the IGMFs suggested by the blazar observations [36-45] ${ }^{11}$.

The fact that the non-helical part of the hypermagnetic fields contribute to the baryon isocurvature perturbation enables us to constrain them at the EWSB, assuming that another mechanism is responsible for the BAU today. In this case, large-scale magnetic fields whose coherence length is larger than the comoving neutron diffusion scale at BBN is permitted, but only tiny field satisfying $B_{0} \lesssim 10^{-18} \mathrm{G}\left(\xi_{0} / 0.0025 \mathrm{pc}\right)^{-1 / 2}$ today, which is well below the lower bound of the IGMFs suggested by the blazar observations. As for the IGMFs with a shorter wave length that have experienced the cascade process, the constraint is almost the same as

\footnotetext{
${ }^{11}$ Especially, if the constraint on the IGMF coherence length suggested in Ref. [45] is correct, it is quite difficult to satisfy both the baryon isocurvature constraints and IGMFs
} 
the case when the baryogenesis from the hypermagnetic helicity decay is responsible for the present BAU. In summary, regardless of its helicity nature, hypermagnetic fields generated before the EWSB cannot be the origin of the IGMFs suggested by the blazar observations. We need another mechanism to generate or amplify the magnetic fields after EWSB. Note that the lower bound of the primordial magnetic fields required as a source of the dynamo amplification of the galactic magnetic fields, $B_{0} \gtrsim 10^{-30} \mathrm{G}$ at $\xi_{0} \gtrsim 10^{2} \mathrm{pc}$ [90], can be satisfied by the primordial hypermagnetic fields generated before the EWSB.

There are several potential uncertainties in our analysis that could change our results. The first is that we have assumed that the symmetric and antisymmetric parts of the power spectrum, $S(q)$ and $A(q)$, are proportional to each other. However, the results are essentially unchanged even if this assumption is relaxed. We see that Eq. (3.5) can be decomposed into contributions from the symmetric and antisymmetric parts as $\overline{S_{B, \mathrm{BBN}}^{2}}=$ $\bar{\eta}_{B}^{-2}\left[\sum\left(\int S S\right.\right.$-like terms $)+\sum\left(\int A A\right.$-like terms $\left.)\right]$, where the symmetric part should be the dominant contribution thanks to the realizability condition. Therefore, by defining an effective helicity fraction, $\epsilon_{\text {eff }}$, by $\epsilon_{\text {eff }}^{2} \equiv \sum\left(\int A A\right.$-like terms $) / \sum\left(\int S S\right.$-like terms $)(\leq 1)$, the constraint is the same as that of $\S 4$.

The second is that the temperature dependence of the weak mixing angle, which can change the efficiency factor of the baryogenesis, $\mathcal{C}$. In our analysis we adopted the value inspired by the one-loop analytic estimate [55], while the fitting formulae for the lattice simulation [35] adopted in Ref. [11] can lead to a smaller value of $\mathcal{C}$. However, in a reasonable choice of the fitting formula, the constraints on the strength and coherence length magnetic fields can be enhanced by a factor of $\mathcal{O}(10)$, but it is not enough for the upper bound from the baryon isocurvature constraints to reach at the lower bound of the IGMFs suggested by the blazar observations, except for the case when the magnetic fields have experienced the MHD cascade with milder decay $\beta=0$ (but see the next discussion). Since there are no accurate calculations on the efficiency factor $\mathcal{C}$ at the sphaleron freezeout $T \simeq 135 \mathrm{GeV}$, we cannot rule out the possibility that it has a much smaller value in reality, but it would be unlikely. If some physics around the EWSB changes the weak mixing angle much more rapidly while keeping the sphaleron rate intact, it would be possible to realize a much smaller value of $\mathcal{C}$ so that small enough baryon isocurvature perturbations and the blazar observations might be simultaneously explained, which is beyond the scope of the present paper.

Finally, the MHD cascade evolution is treated in a relatively simplified way. We evaluate the eddy turnover scale (Eq. (3.13)) assuming the equipartition of the magnetic and fluid kinetic energy, which leads to $v \simeq v_{\mathrm{A}}$. If the eddy turnover scale is smaller, we might have a stronger IGMF today but with a smaller coherence length. In order for the remnant magnetic fields to reach at the lower bound of the IGMFs suggested by blazars, however, we need the changes of the eddy turnover scale with more than a few orders of magnitude, which would be unlikely. Moreover, the upper bound of the IGMF strength in our analysis is obtained in the case with the cascade decay parameter $\beta=0$ at very tiny helicity. Evolution with $\beta=0$ is the case of the so-called inverse cascade with the maximally helical magnetic fields, which is guaranteed by the helicity conservation. We do not rule out such a possibility, but do not expect the evolution $\beta \simeq 0$ can be realized for the non-maximally helical magnetic fields, where there are no conserved quantity to support the evolution. In summary, although there still remain several uncertainties in our analysis, we conclude that they do not change our results significantly.

What we have presented in this paper gives essential constraints on the model building of magnetogenesis. As we have shown that the strength of the IGMFs suggested by the blazar 
observations is well above the baryon isocurvature constraints, any attempts to explain it by a primordial magnetic fields before the EWSB is ruled out. For example, a first order phase transition in the physics beyond the SM at a higher energy scale than the EWSB does not work as a way to explain the origin of IGMFs. Hypermagnetic fields just to explain the BAU are still allowed, but the helicity fraction should not be too small, $\epsilon_{\mathrm{fo}} \gtrsim 10^{-8}$. There has been a hope to test the scenario by IGMF observations, but we conclude that main part of the observationally suggested IGMFs should be generated or amplified after the EWSB, while magnetic fields responsible for the BAU is subdominant contribution of them. In order to test the scenario, we need to develop the way to remove such "foreground" IGMFs. ${ }^{12}$

\section{Acknowledgments}

The work of FU is supported by the Forefront Physics and Mathematics Program to Drive Transformation (FoPM). This work was partially supported by JSPS KAKENHI Grant-inAid for Scientific Research (C) JP19K03842 (KK), Scientific Research (S) 20H05639(JY), Innovative Area 19H04610(KK), and 20H05248(JY).

\section{A Gauge choice}

Since the source term (2.3) is gauge dependent without taking a spatial average, we have to specify a gauge to carry out our calculation. In the main text of the present paper, we have imposed the Coulomb gauge condition, $\boldsymbol{\nabla} \cdot \boldsymbol{A}_{\mathcal{A}}(\boldsymbol{x})=0$. In this Appendix, we see that we can determine the power spectrum of the vector potential, $\boldsymbol{A}_{\mathcal{A}}$, as we do in $\S 2.2$, by choosing the Coulomb gauge.

As for the magnetic field, $\boldsymbol{B}_{\mathcal{A}}$, we can assume the homogeneity and the isotropy, according to the cosmological principle. Because of the homogeneity of the universe, the two-point correlation function of the magnetic fields can be written as a function of the relative coordinate.

$$
\left\langle B_{\mathcal{A} i}(\boldsymbol{x}) B_{\mathcal{A} j}(\boldsymbol{x}+\boldsymbol{r})\right\rangle=\tilde{\mathcal{F}}_{i j}^{B}(\boldsymbol{r}) .
$$

Transforming this equation into the wavenumber space, we see that the power spectrum of the magnetic field is given by $\tilde{\mathcal{F}}_{i j}^{B}(\boldsymbol{k})$, the Fourier transform of $\tilde{\mathcal{F}}_{i j}^{B}(\boldsymbol{x})$.

$$
\left\langle B_{\mathcal{A} i}^{*}(\boldsymbol{k}) B_{\mathcal{A} j}\left(\boldsymbol{k}^{\prime}\right)\right\rangle=(2 \pi)^{3} \delta^{3}\left(\boldsymbol{k}-\boldsymbol{k}^{\prime}\right) \tilde{\mathcal{F}}_{i j}^{B}(\boldsymbol{k}) .
$$

Notice that the factor $\delta^{3}\left(\boldsymbol{k}-\boldsymbol{k}^{\prime}\right)$ appearing in the left hand side of the equation is a consequence of the homogeneity. It immediately follows from Eq. (A.2) that $\tilde{\mathcal{F}}_{j i}^{B *}(\boldsymbol{k})=\tilde{\mathcal{F}}_{i j}^{B}(\boldsymbol{k})$. Because of the isotropy and the divergenceless property of the magnetic field in addition to this, the power spectrum of the magnetic field is characterized by two real-valued functions, $\tilde{S}^{B}(k)$ and $\tilde{A}^{B}(k)$ as

$$
\tilde{\mathcal{F}}_{i j}^{B}(\boldsymbol{k})=P_{i j}(\hat{\boldsymbol{k}}) \tilde{S}^{B}(k)+i \epsilon_{i j m} \hat{k}_{m} \tilde{A}^{B}(k), \quad P_{i j}(\hat{\boldsymbol{k}})=\delta_{i j}-\hat{k}_{i} \hat{k}_{j} .
$$

As for the vector potential, $\boldsymbol{A}_{\mathcal{A}}$, whether $\left\langle A_{\mathcal{A} i}^{*}(\boldsymbol{k}) A_{\mathcal{A} j}\left(\boldsymbol{k}^{\prime}\right)\right\rangle$ has the factor $\delta^{3}\left(\boldsymbol{k}-\boldsymbol{k}^{\prime}\right)$ depends on the choice of gauge. The reason why a gauge transformation breaks the condition

\footnotetext{
${ }^{12}$ Another option is to explain the blazar observation by some ways other than the IGMFs such as the beam plasma instability [91].
} 
equivalent to homogeneity is that values of the vector potential are not physical ones and that the homogeneity of the universe does not necessarily require the homogeneity of the vector potential field. However, with the Coulomb gauge condition, the homogeneity is maintained, and we can define the power spectrum of the vector potential, ${ }^{13}$

$$
\left\langle A_{\mathcal{A} i}^{*}(\boldsymbol{k}) A_{\mathcal{A} j}\left(\boldsymbol{k}^{\prime}\right)\right\rangle=:(2 \pi)^{3} \delta^{3}\left(\boldsymbol{k}-\boldsymbol{k}^{\prime}\right) \tilde{\mathcal{F}}_{i j}^{A}(\boldsymbol{k}) .
$$

Since $\boldsymbol{B}_{\mathcal{A}}=\boldsymbol{\nabla} \times \boldsymbol{A}_{\mathcal{A}}$, the power spectra, $\tilde{\mathcal{F}}_{i j}^{B}$ and $\tilde{\mathcal{F}}_{i j}^{A}$, are related via

$$
\tilde{\mathcal{F}}_{i j}^{B}(\boldsymbol{k})=\epsilon_{i l m} \epsilon_{j r s} k_{l} k_{r} \tilde{\mathcal{F}}_{m s}^{A}(\boldsymbol{k}) .
$$

Similarly to the case of magnetic field, $\tilde{\mathcal{F}}_{j i}^{A *}(\boldsymbol{k})=\tilde{\mathcal{F}}_{i j}^{A}(\boldsymbol{k})$, the Coulomb gauge condition $k_{i} \tilde{\mathcal{F}}_{i j}^{A}(\boldsymbol{k})=0$, and the isotropy of the magnetic field leaves only two real-valued functions, $\tilde{S}(k), \tilde{A}(k)$, as the degrees of freedom of the power spectrum of the vector potential,

$$
\tilde{\mathcal{F}}_{i j}^{A}(\boldsymbol{k})=P_{i j}(\hat{\boldsymbol{k}}) \tilde{S}(k)+i \epsilon_{i j m} \hat{k}_{m} \tilde{A}(k) .
$$

Using Eq. (A.5), $\tilde{S}^{B}, \tilde{A}^{B}$ and $\tilde{S}, \tilde{A}$ are related via

$$
\tilde{S}^{B}(k)=k^{2} \tilde{S}(k), \quad \tilde{A}^{B}(k)=k^{2} \tilde{A}(k) .
$$

In addition to this, as to the one-point function, because of the Coulomb gauge condition,

$$
\left\langle A_{\mathcal{A} i}(\boldsymbol{k})\right\rangle=0
$$

follows from the homogeneity of the magnetic field, $\left\langle B_{\mathcal{A} i}(\boldsymbol{k})\right\rangle=0 .{ }^{14}$

The physical meaning of symmetric and antisymmetric parts of the power spectrum becomes clearer if we use the popular notations

$$
E_{\mathrm{M}}(k)=\frac{k^{4}}{2 \pi^{2}} \tilde{S}(k), \quad H_{\mathrm{M}}(k)=\frac{k^{3}}{\pi^{2}} \tilde{A}(k) .
$$

An integration of $E_{\mathrm{M}}(k)$ over $k$ gives the energy density of the fields, $\mathcal{E}=\int d k E_{\mathrm{M}}(k)$, and an integration of $H_{\mathrm{M}}(k)$ the helicity density, $h=\int d k H_{\mathrm{M}}(k)$. Therefore, the symmetric part of the power spectrum determines the energy of the fields, while the antisymmetric part determines the magnetic helicity of the fields. One can explicitly see this property from Eqs. (2.15) and (2.16) as well.

\section{B Correlation functions in the position space}

In this appendix, we rederive the correlation function of the baryon isocurvature perturbation $\mathcal{G}$, defined in Eq. (2.24), in the real coordinate space. ${ }^{15}$

\footnotetext{
${ }^{13}$ We see this fact from $P_{i a}(\hat{\boldsymbol{k}}) P_{j b}\left(\hat{\boldsymbol{k}}^{\prime}\right) \epsilon_{a l r} \epsilon_{b m s}\left\langle B_{\mathcal{A} l}^{*}(\boldsymbol{k}) B_{\mathcal{A} m}\left(\boldsymbol{k}^{\prime}\right)\right\rangle=P_{i a}(\hat{\boldsymbol{k}}) P_{j b}\left(\hat{\boldsymbol{k}}^{\prime}\right) k_{r} k_{s}^{\prime}\left\langle A_{\mathcal{A} a}^{*}(\boldsymbol{k}) A_{\mathcal{A} b}\left(\boldsymbol{k}^{\prime}\right)\right\rangle=$ $k_{r} k_{s}^{\prime}\left\langle A_{\mathcal{A} i}^{*}(\boldsymbol{k}) A_{\mathcal{A} j}\left(\boldsymbol{k}^{\prime}\right)\right\rangle \propto \delta^{3}\left(\boldsymbol{k}-\boldsymbol{k}^{\prime}\right)$, with the second equality valid under the Coulomb gauge condition, $P_{i j}(\hat{\boldsymbol{k}}) A_{\mathcal{A} j}(\boldsymbol{k})=A_{\mathcal{A} i}(\boldsymbol{k})$.

${ }^{14}$ We see this from $-i P_{i a}(\hat{\boldsymbol{k}}) \epsilon_{a l r}\left\langle B_{\mathcal{A l}}(\boldsymbol{k})\right\rangle=P_{i a}(\hat{\boldsymbol{k}}) k_{r}\left\langle A_{\mathcal{A} a}(\boldsymbol{k})\right\rangle=k_{r}\left\langle A_{\mathcal{A} i}(\boldsymbol{k})\right\rangle=0$, with the second equality valid under the Coulomb gauge condition.

${ }^{15}$ Since we already have an expression in the wavenumber space, we can just Fourier transform it. However, here we work in the position space from the beginning. This reformulation will be helpful to intuitively understand how the baryon isocurvature perturbation is generated. See Ref. [7] for the earlier work about the procedure.
} 
Power spectrum in the position space We define three functions $F_{1}, F_{2}, F_{3}$, that characterize the power spectrum of the magnetic field in the position space,

$$
F_{1}(R, t) \delta_{i j}+F_{2}(R, t) R^{i} R^{j} \equiv \int \frac{d^{3} q}{(2 \pi)^{3}} e^{+i \boldsymbol{q} \cdot \boldsymbol{R}} P_{i j}(\hat{\boldsymbol{q}}) S(q),
$$

or equivalently

$$
\begin{aligned}
& F_{1}(R)=\frac{1}{2 \pi^{2}} \int d(\ln q)\left[\left(-\frac{1}{R^{3}}+\frac{q^{2}}{R}\right) \sin (q R)+\frac{q}{R^{2}} \cos (q R)\right] S(q), \\
& F_{2}(R)=\frac{1}{2 \pi^{2}} \int d(\ln q)\left[\left(\frac{3}{R^{5}}-\frac{q^{2}}{R^{3}}\right) \sin (q R)-\frac{3 q}{R^{4}} \cos (q R)\right] S(q),
\end{aligned}
$$

and

$$
F_{3}(\boldsymbol{R}, t) \equiv \int \frac{d^{3} q}{(2 \pi)^{3}} e^{+i \boldsymbol{q} \cdot \boldsymbol{R}} A(q)
$$

Here we have introduced a characteristic scale $k_{\sigma}$ that normalizes the dimensionful parameters, such as $\boldsymbol{q} \equiv \boldsymbol{k} / k_{\sigma}$ and $\boldsymbol{R} \equiv k_{\sigma} \boldsymbol{x}$. $S(q)$ and $A(q)$ are also normalized by another parameter $B$, which characterizes the magnetic field strength so that the magnetic energy and helicity are evaluated as $\mathcal{E} \propto B^{2}$ and $h \propto B^{2} / k_{\sigma}$, respectively. Then the power spectrum $\mathcal{F}_{i j}^{A}$ in the position space can be written in terms of these functions as

$$
\mathcal{F}_{i j}^{A}(\boldsymbol{R})=F_{1}(R) \delta_{i j}+F_{2}(R) R^{i} R^{j}+\epsilon_{i j m} F_{3, m}(\boldsymbol{R}),
$$

where $F_{3, m}(\boldsymbol{R}) \equiv \frac{\partial}{\partial R^{m}} F_{3}(\boldsymbol{R}) . \quad F_{1}$ and $F_{2}$ characterizes the symmetric part of the two-point correlation function of the magnetic field, and $F_{3}$ does its antisymmetric part. We shall note that $F_{3, m}$ has the following properties. By choosing the coordinate system so that the $\boldsymbol{R}$ lies in the $\boldsymbol{e}_{3}$ direction, or $\boldsymbol{e}_{3} \| \boldsymbol{R}$, for the coordinate $\boldsymbol{e}_{1,2}$, which are perpendicular to $\boldsymbol{e}_{3}$, $F_{3, m}$ vanishes because the integrand of (B.4) is odd with respect to $q_{3}$. Or we shall write $F_{3, i}(\boldsymbol{R})=0$ for $i=1,2$. On the other hand, for the coordinate $\boldsymbol{e}_{3}, F_{3,3}$ gives finite value as

$$
F_{3,3}(\boldsymbol{R})=\frac{1}{2 \pi^{2} R^{2}} \int d q[q R \cos (q R)-\sin (q R)] A(q) \equiv F_{3,3}(R, t),
$$

as a function of the absolute value $R$. As long as $S(q)$ as well as $A(q)$ are localized at the scale $q \sim 1 \Leftrightarrow k \sim k_{\sigma}^{-1}, F_{1,2,3}(R)$ are also peaked at $R \sim 1 \Leftrightarrow r \sim k_{\sigma}^{-1}$. Note also that the net mean baryon asymmetry is given from Eq. (2.5) in terms of $F_{3,3}$ as

$$
\bar{\eta}_{B}=-\left.6 \mathcal{C} k_{\sigma}^{-1} B^{2} F_{3,3}^{\prime}(0)\right|_{T=T_{\mathrm{fo}}},
$$

where the prime denotes the derivative with respect to $R$. Particularly in the coordinate coordinate system with $\boldsymbol{e}_{i, j}$ with $i, j=1,2$ being perpendicular to $\boldsymbol{R}$ whereas $\boldsymbol{e}_{3}$ is parallel to $\boldsymbol{R}$ so that $\boldsymbol{e}_{1}, \boldsymbol{e}_{2}$, and $\boldsymbol{e}_{3}$ construct the right-handed coordinate system (therefore $R^{i}=0$ for $i=1,2$ and $R^{3}=R$.), the Fourier transform of the power spectrum that contains the anti-symmetric part as

$$
\mathcal{F}_{i j}^{A}(\boldsymbol{R})=\int \frac{d^{3} q}{(2 \pi)^{3}} e^{+i \boldsymbol{q} \boldsymbol{R}} \mathcal{F}_{i j}^{A}(\boldsymbol{q})=F_{1}(R) \delta_{i j}+\epsilon_{i j} F_{3}(R),
$$

where $\epsilon_{i j}$ is the 2-dimensional Levi-Civita tensor with $\epsilon_{12}=1$. We shall note that other components of the magnetic field power spectrum are given as

$$
\mathcal{F}_{i 3}^{A}(\boldsymbol{R})=\mathcal{F}_{3 i}^{A}(\boldsymbol{R})=0, \quad \mathcal{F}_{33}^{A}(\boldsymbol{R})=F_{1}(R)+F_{2}(R) R^{2} .
$$


Baryon isocurvature perturbation in the position space Let us now derive the expressions of the baryon isocurvature perturbations in terms of $F_{i}$ s. From Eq. (2.22), the two-point correlation function of the baryon isocurvature perturbation, $\mathcal{G}(\boldsymbol{R})$, defined in Eq. (2.24), is proportional to a four-point function of the vector potential as

$$
\begin{aligned}
&\langle(\boldsymbol{A} \cdot \nabla \times \boldsymbol{A})(\boldsymbol{x})(\boldsymbol{A} \cdot \nabla \times \boldsymbol{A})(\boldsymbol{x}+\boldsymbol{r})\rangle \\
&=\epsilon_{i j k} \epsilon_{l m n} \lim _{\boldsymbol{s}, \boldsymbol{t} \rightarrow 0} \frac{\partial}{\partial s^{i}} \frac{\partial}{\partial t^{l}}\left\langle A_{k}(\boldsymbol{x}) A_{j}(\boldsymbol{x}+\boldsymbol{s}) A_{n}(\boldsymbol{x}+\boldsymbol{r}) A_{m}(\boldsymbol{x}+\boldsymbol{r}+\boldsymbol{t})\right\rangle \\
&=\epsilon_{i j k} \epsilon_{l m n} \lim _{\boldsymbol{s}, \boldsymbol{t} \rightarrow 0} \frac{\partial}{\partial s^{i}} \frac{\partial}{\partial t^{l}}[\left\langle A_{k}(\boldsymbol{x}) A_{j}(\boldsymbol{x}+\boldsymbol{s})\right\rangle\left\langle A_{n}(\boldsymbol{x}+\boldsymbol{r}) A_{m}(\boldsymbol{x}+\boldsymbol{r}+\boldsymbol{t})\right\rangle \\
&+\left\langle A_{k}(\boldsymbol{x}) A_{n}(\boldsymbol{x}+\boldsymbol{r})\right\rangle\left\langle A_{j}(\boldsymbol{x}+\boldsymbol{s}) A_{m}(\boldsymbol{x}+\boldsymbol{r}+\boldsymbol{t})\right\rangle \\
&\left.+\left\langle A_{k}(\boldsymbol{x}) A_{m}(\boldsymbol{x}+\boldsymbol{r}+\boldsymbol{t})\right\rangle\left\langle A_{n}(\boldsymbol{x}+\boldsymbol{r}) A_{j}(\boldsymbol{x}+\boldsymbol{s})\right\rangle\right] \\
&=\epsilon_{i j k} \epsilon_{l m n} \lim _{\boldsymbol{s}, \boldsymbol{t} \rightarrow 0} \frac{\partial}{\partial s^{i}} \frac{\partial}{\partial t^{l}}[ \mathcal{F}_{k j}^{A}\left(k_{\sigma} \boldsymbol{s}\right) \mathcal{F}_{n m}^{A}\left(k_{\sigma} \boldsymbol{t}\right)+\mathcal{F}_{k n}^{A}\left(k_{\sigma} \boldsymbol{r}\right) \mathcal{F}_{j m}^{A}\left(k_{\sigma}(\boldsymbol{r}-\boldsymbol{s}+\boldsymbol{t})\right) \\
&\left.+\mathcal{F}_{k m}^{A}\left(k_{\sigma}(\boldsymbol{r}+\boldsymbol{t})\right) \mathcal{F}_{j n}^{A}\left(k_{\sigma}(\boldsymbol{r}-\boldsymbol{s})\right)\right] \times k_{\sigma}^{-4} B^{4}
\end{aligned}
$$

In the second equality, we decomposed the four-point function into pairs of two-point functions, assuming the Gaussianity of the stochasticity. In the last equality we have used the fact that the ensemble average $\left\langle A_{i}(\boldsymbol{x}) A_{j}(\boldsymbol{x}+\boldsymbol{r})\right\rangle$ is $\boldsymbol{x}$-independent, and hence $\left\langle A_{i}(\boldsymbol{x}) A_{j}(\boldsymbol{x}+\boldsymbol{r})\right\rangle=$ $(1 / V) \int d^{3} x\left\langle A_{i}(\boldsymbol{x}) A_{j}(\boldsymbol{x}+\boldsymbol{r})\right\rangle=k_{\sigma}^{-2} B^{2} \mathcal{F}_{i j}^{A}\left(k_{\sigma} \boldsymbol{r}\right)$. Each contribution in Eq. (B.10) is calculated in terms of the functions $F_{i}$ s as

$$
\begin{aligned}
& \epsilon_{i j k} \epsilon_{l m n} \lim _{\boldsymbol{s , t \rightarrow 0}} \frac{\partial}{\partial s^{i}} \frac{\partial}{\partial t^{l}}\left(\mathcal{F}_{k j}^{A}\left(k_{\sigma} s\right) \mathcal{F}_{n m}^{A}\left(k_{\sigma} \boldsymbol{t}\right)\right)=36 k_{\sigma}^{2} F_{3,3}^{\prime 2}(0), \\
& \epsilon_{i j k} \epsilon_{l m n} \lim _{\boldsymbol{s , t \rightarrow 0}} \frac{\partial}{\partial s^{i}} \frac{\partial}{\partial t^{l}}\left(\mathcal{F}_{k n}^{A}\left(k_{\sigma} \boldsymbol{r}\right) \mathcal{F}_{j m}^{A}\left(k_{\sigma}(\boldsymbol{r}-\boldsymbol{s}+\boldsymbol{t})\right)\right) \\
& =2 k_{\sigma}^{2}\left[-\frac{2}{R} F_{1} F_{1}^{\prime}-F_{1} F_{1}^{\prime \prime}+3 F_{1} F_{2}+R F_{1} F_{2}^{\prime}-R F_{1}^{\prime} F_{2}+R^{2} F_{2}^{2}-F_{3,3}\left(F_{3,3}^{\prime \prime}+\frac{2}{R} F_{3,3}^{\prime}-\frac{2}{R^{2}} F_{3,3}\right)\right],
\end{aligned}
$$

$$
\begin{aligned}
& \epsilon_{i j k} \epsilon_{l m n} \lim _{\boldsymbol{s , t \rightarrow 0}} \frac{\partial}{\partial s^{i}} \frac{\partial}{\partial t^{l}}\left(\mathcal{F}_{k m}^{A}\left(k_{\sigma}(\boldsymbol{r}+\boldsymbol{t})\right) \mathcal{F}_{j n}^{A}\left(k_{\sigma}(\boldsymbol{r}-\boldsymbol{s})\right)\right) \\
& =2 k_{\sigma}^{2}\left(F_{1}^{\prime 2}+R^{2} F_{2}^{2}-2 R F_{1}^{\prime} F_{2}+F_{3}^{\prime 2}+\frac{2}{R} F_{3,3} F_{3,3}^{\prime}+\frac{3}{R^{2}} F_{3,3}^{2}\right) .
\end{aligned}
$$

Here we have omitted the arguments of the functions $F_{i}$ otherwise stated. Note that the final result is independent of the choice of the coordinate system.

The detailed calculations to obtain Eq. (B.11a), (B.11a), and (B.11c) are summarized in what follows. We adopt the coordinate system such that the $\boldsymbol{R}$ lies in the $\boldsymbol{e}_{3}$ direction and 
then can use Eqs. (B.8), (B.9). First let us compute derivatives of $F_{3,3}$.

$$
\begin{aligned}
F_{3,311}(\boldsymbol{R}) & =F_{3,322}(\boldsymbol{R})=\frac{F_{3,3}(R)}{R}, \quad F_{3,333}(\boldsymbol{R})=F_{3,3}^{\prime}(R), \\
\delta_{i j} F_{3,3 i j}(\boldsymbol{R}) & =-\int \frac{d q}{2 \pi^{2}} \frac{q^{2}}{R} \sin (q R) A(q)=F_{3,3}^{\prime}(R)+\frac{2 F_{3,3}(R)}{R}, \\
\delta_{i j} F_{3,3 m i j}(\boldsymbol{R}) & =-\delta_{3 m} \int \frac{d q}{2 \pi^{2}} \frac{q^{2}}{R^{2}}(q R \cos (q R)-\sin (q R)) A(q) \\
& =\delta_{3 m}\left(F_{3,3}^{\prime \prime}(R)+\frac{2 F_{3,3}^{\prime}(R)}{R}-\frac{2 F_{3,3}(R)}{R^{2}}\right),
\end{aligned}
$$

where subscripts denote partial derivatives, e.g., $F_{3,3 i j}(\boldsymbol{R}) \equiv \frac{\partial^{2}}{\partial R^{j} \partial R^{i}} R_{3,3}(R)$. Now Eq. (B.11a) is calculated as

$$
\begin{aligned}
& \epsilon_{i j k} \epsilon_{l m n} \lim _{\boldsymbol{s}, \boldsymbol{t} \rightarrow 0} \frac{\partial}{\partial s^{i}} \frac{\partial}{\partial t^{l}}\left(\mathcal{F}_{k j}^{A}\left(k_{\sigma} s\right) \mathcal{F}_{n m}^{A}\left(k_{\sigma} \boldsymbol{t}\right)\right)=\left(\epsilon_{i j k} \lim _{\boldsymbol{s} \rightarrow 0} \frac{\partial}{\partial s^{i}} \mathcal{F}_{k j}^{A}\left(k_{\sigma} s\right)\right)^{2} \\
&=\left(-2 \delta_{i m} \lim _{\boldsymbol{s} \rightarrow 0} \frac{\partial}{\partial s^{i}} F_{3,3 m}\left(k_{\sigma} s\right)\right)^{2}=36 k_{\sigma}^{2} F_{3,3}^{\prime 2}(0) .
\end{aligned}
$$

On the calculation of Eqs. (B.11b) and (B.11c), let us note that

$$
\frac{\partial}{\partial R^{j}} F_{i}(R)=\frac{R^{j}}{R} F_{i}^{\prime}(R), \quad \frac{\partial}{\partial R^{j}} \frac{\partial}{\partial R^{m}} F_{i}(R)=\frac{R^{j} R^{m}}{R^{2}}\left(F_{i}^{\prime \prime}(R)-\frac{F_{i}^{\prime}}{R}\right)+\frac{F_{i}^{\prime}}{R} \delta_{j m},
$$

for $i=1,2$, and hence

$$
\delta_{j m} \frac{\partial}{\partial R^{j}} \frac{\partial}{\partial R^{m}} F_{i}(R)=F_{i}^{\prime \prime}(R)+\frac{2 F_{i}^{\prime}(R)}{R} .
$$

The first term in Eq. (B.16) is non-vanishing only for $j=3$ so that

$$
\frac{\partial}{\partial R^{j}} F_{i}(R)=F_{i}^{\prime}(R) \delta_{j 3}, \quad \frac{\partial}{\partial R^{3}} \frac{\partial}{\partial R^{3}} F_{i}(R)=F_{i}^{\prime \prime}(R) .
$$

By using these expressions, we reach the expression Eq. (B.11b) as

$$
\begin{aligned}
& \epsilon_{i j k} \epsilon_{l m n} \lim _{\boldsymbol{s}, \boldsymbol{t} \rightarrow 0} \frac{\partial}{\partial s^{i}} \frac{\partial}{\partial t^{l}}\left(\mathcal{F}_{k n}^{A}\left(k_{\sigma} \boldsymbol{r}\right) \mathcal{F}_{j m}^{A}\left(k_{\sigma}(\boldsymbol{r}-\boldsymbol{s}+\boldsymbol{t})\right)\right) \\
& =\epsilon_{i j k} \epsilon_{l m n} k_{\sigma}^{2}\left(F_{1}(R) \delta_{k n}+F_{2}(R) R_{k} R_{n}+\epsilon_{k n s} F_{3,3 s}(R)\right) \\
& \quad \times \lim _{\boldsymbol{S}, \boldsymbol{T} \rightarrow 0} \frac{\partial}{\partial S^{i}} \frac{\partial}{\partial T^{l}}\left[F_{1}(|\boldsymbol{R}-\boldsymbol{S}+\boldsymbol{T}|) \delta_{j m}+F_{2}(|\boldsymbol{R}-\boldsymbol{S}+\boldsymbol{T}|)\left(R_{j}-S_{j}+T_{j}\right)\left(R_{m}-S_{m}+T_{m}\right)\right. \\
& \left.=2 k_{\sigma}^{2}\left[-\frac{R}{R}-\boldsymbol{S}+\boldsymbol{T}\right)\right]
\end{aligned}
$$


where we have defined as $\boldsymbol{S} \equiv k_{\sigma} s$ and $\boldsymbol{T} \equiv k_{\sigma} \boldsymbol{t}$. In the same way, Eq. (B.11c) can be derived as

$$
\begin{aligned}
\epsilon_{i j k} \epsilon_{l m n} \lim _{\boldsymbol{s}, \boldsymbol{t} \rightarrow 0} \frac{\partial}{\partial s^{i}} \frac{\partial}{\partial t^{l}}\left(\mathcal{F}_{k m}^{A}\left(k_{\sigma}(\boldsymbol{r}+\boldsymbol{t})\right) \mathcal{F}_{j n}^{A}\left(k_{\sigma}(\boldsymbol{r}-\boldsymbol{s})\right)\right) \\
=\epsilon_{i j k} \epsilon_{l m n} k_{\sigma}^{2} \lim _{\boldsymbol{s}, \boldsymbol{t} \rightarrow 0} \frac{\partial}{\partial T^{l}}\left[F_{1}(|\boldsymbol{R}+\boldsymbol{T}|) \delta_{k m}+F_{2}(|\boldsymbol{R}+\boldsymbol{T}|)\left(R_{k}+T_{k}\right)\left(R_{m}+T_{m}\right)+\epsilon_{k m w} F_{3,3 w}(\boldsymbol{R}+\boldsymbol{T})\right] \\
\times \frac{\partial}{\partial S^{i}}\left[F_{1}(|\boldsymbol{R}-\boldsymbol{S}|) \delta_{j n}+F_{2}(|\boldsymbol{R}-\boldsymbol{S}|)\left(R_{j}-S_{j}\right)\left(R_{n}-S_{n}\right)+\epsilon_{j n z} F_{3,3 z}(\boldsymbol{R}-\boldsymbol{S})\right] \\
=2 k_{\sigma}^{2}\left[F_{1}^{\prime 2}+R^{2} F_{2}^{2}-2 R F_{1}^{\prime} F_{2}+F_{3}^{\prime 2}+\frac{2}{R} F_{3,3} F_{3,3}^{\prime}+\frac{3}{R^{2}} F_{3,3}^{2}\right] .
\end{aligned}
$$

In summary, we obtain the two-point correlation function of the helicity in terms of the power spectrum as

$$
\begin{aligned}
\langle(\boldsymbol{A} \cdot \boldsymbol{\nabla} \times \boldsymbol{A})(\boldsymbol{x})(\boldsymbol{A} \cdot \boldsymbol{\nabla} \times \boldsymbol{A})(\boldsymbol{x}+\boldsymbol{r})\rangle-\langle(\boldsymbol{A} \cdot \boldsymbol{\nabla} \times \boldsymbol{A})(\boldsymbol{x})\rangle^{2} & \\
=2 k_{\sigma}^{-2} B^{4}\left[F_{1}^{\prime 2}-\frac{2}{R} F_{1} F_{1}^{\prime}-F_{1} F_{1}^{\prime \prime}+3 F_{1} F_{2}\right. & +R F_{1} F_{2}^{\prime}-3 R F_{1}^{\prime} F_{2}+2 R^{2} F_{2}^{2} \\
& \left.+F_{3,3}^{\prime 2}-F_{3,3}\left(F_{3,3}^{\prime \prime}-\frac{5}{R^{2}} F_{3,3}\right)\right] .
\end{aligned}
$$

We can easily check that Eq. (B.21) is consistent with the one given in Ref. [7]. Since in Ref. [7] the magnetic fields are non-helical, $A(q)=0$, postulating that another mechanism is responsible for the BAU today, we have additional contributions from the helical part $A(q)$, which appear in terms of $F_{3,3}$.

Now we are ready to evaluate the baryon isocurvature perturbations in terms of the magnetic field power spectrum. From Eqs. (2.22), (B.7), (B.21) we obtain

$$
\mathcal{G}(\boldsymbol{R})=\frac{\mathcal{C}^{2}}{\bar{\eta}_{B}^{2}}\langle(\boldsymbol{A} \cdot \boldsymbol{\nabla} \times \boldsymbol{A})(\boldsymbol{x})(\boldsymbol{A} \cdot \boldsymbol{\nabla} \times \boldsymbol{A})(\boldsymbol{x}+\boldsymbol{r})\rangle-\left.\langle(\boldsymbol{A} \cdot \boldsymbol{\nabla} \times \boldsymbol{A})(\boldsymbol{x})\rangle^{2}\right|_{T=T_{\mathrm{fo}}}=\frac{\tilde{G}(R)}{18 F_{3,3}^{\prime 2}(0)},
$$

with $R \equiv k_{\sigma}|\boldsymbol{r}|$ and

$$
\begin{aligned}
\tilde{G}(R) \equiv & F_{1}^{\prime 2}(R)-\frac{2}{R} F_{1}(R) F_{1}^{\prime}(R)-F_{1}(R) F_{1}^{\prime \prime}(R)+3 F_{1}(R) F_{2}(R)+R F_{1}(R) F_{2}^{\prime}(R) \\
& -3 R F_{1}^{\prime}(R) F_{2}(R)+2 R^{2} F_{2}^{2}(R)+F_{3,3}^{\prime 2}(R)-F_{3,3}(R)\left(F_{3,3}^{\prime \prime}(R)-\frac{5}{R^{2}} F_{3,3}(R)\right) .
\end{aligned}
$$

Delta function spectrum In the case of the delta-function model, $S(q)$ and $A(q)$,

$$
S(q)=\delta(q-1), \quad A(q)=\epsilon \delta(q-1),
$$

$F_{i}$ s become

$$
\begin{aligned}
F_{1}(R) & =\frac{1}{2 \pi^{2}}\left[\left(\frac{1}{R}-\frac{1}{R^{3}}\right) \sin R+\frac{1}{R^{2}} \cos R\right], \\
F_{2}(R) & =\frac{1}{2 \pi^{2}}\left[\left(-\frac{1}{R^{3}}+\frac{3}{R^{5}}\right) \sin R-\frac{3}{R^{4}} \cos R\right], \\
F_{3,3}(R) & =\frac{\epsilon}{2 \pi^{2}}\left[-\frac{1}{R^{2}} \sin R+\frac{1}{R} \cos R\right] .
\end{aligned}
$$


Then inserting them into Eq. (B.23), we can compute the correlator of baryon isocurvature perturbations as

$$
\mathcal{G}(\boldsymbol{R})=\frac{1}{4}\left(\frac{1}{\epsilon^{2}}+1\right)\left[\frac{2}{R^{2}}+\frac{2}{R^{4}}+\frac{3}{R^{6}}-\frac{6}{R^{5}} \sin 2 R+\left(\frac{4}{R^{4}}-\frac{3}{R^{6}}\right) \cos 2 R\right] .
$$

Power-law-like spectrum In the case of the power law with exponential cutoff model, $S(q)$ and $A(q)$,

$$
S(q)=q^{\alpha} \exp \left(-q^{2}\right), \quad A(q)=\epsilon q^{\alpha} \exp \left(-q^{2}\right),
$$

$F_{i}$ s become

$$
\begin{aligned}
F_{1}(R) & =\frac{\Gamma\left(\frac{3+\alpha}{2}\right)}{60 \pi^{2}}\left[10 F\left(\frac{3+\alpha}{2}, \frac{5}{2},-\frac{R^{2}}{4}\right)-\frac{3+\alpha}{2} F\left(\frac{5+\alpha}{2}, \frac{7}{2},-\frac{R^{2}}{4}\right) R^{2}\right], \\
F_{2}(R) & =\frac{\Gamma\left(\frac{5+\alpha}{2}\right)}{60 \pi^{2}} F\left(\frac{5+\alpha}{2}, \frac{7}{2},-\frac{R^{2}}{4}\right), \\
F_{3,3}(R) & =\frac{\Gamma\left(1+\frac{\alpha}{2}\right) \epsilon}{4 \pi^{2}}\left[F\left(1+\frac{\alpha}{2}, \frac{1}{2},-\frac{R^{2}}{4}\right)-F\left(1+\frac{\alpha}{2}, \frac{3}{2},-\frac{R^{2}}{4}\right)\right] \frac{1}{R},
\end{aligned}
$$

where $F(a, c, z)$ is the confluent hypergeometric function. Then inserting them into Eq. (B.23), we can compute the correlator of baryon isocurvature perturbations. The asymptotic behavior in the limit $R \rightarrow 0, \mathcal{G}(\boldsymbol{R})$ is

$$
\mathcal{G}(\boldsymbol{R})=\frac{1}{3}\left[\frac{\Gamma\left(\frac{3+\alpha}{2}\right) \Gamma\left(\frac{5+\alpha}{2}\right)}{\Gamma\left(2+\frac{\alpha}{2}\right)^{2} \epsilon^{2}}+1\right]-\frac{1}{18}\left[\frac{\Gamma\left(\frac{3+\alpha}{2}\right) \Gamma\left(\frac{7+\alpha}{2}\right)}{\Gamma\left(2+\frac{\alpha}{2}\right)^{2} \epsilon^{2}}+2+\frac{\alpha}{2}\right] R^{2}+\mathcal{O}\left(R^{4}\right)
$$

while in the opposite limit $R \rightarrow \infty$,

$$
\begin{array}{r}
\mathcal{G}(\boldsymbol{R})=\frac{\Gamma(3+\alpha)^{2}}{\Gamma\left(2+\frac{\alpha}{2}\right)^{2}}\left[\frac{1}{\epsilon^{2}}(3+\alpha)(5+\alpha) \frac{-1+\cos (\pi \alpha)}{\alpha(2+\alpha)^{2}}+(2-\alpha) \frac{1+\cos (\pi \alpha)}{(1+\alpha)^{2}}\right] R^{-8-2 \alpha} \\
+\mathcal{O}\left(R^{-10-2 \alpha}\right)
\end{array}
$$

Comparison of models Finally, let us compare the behaviors of the correlation function of the baryon isocurvature perturbation for the delta-function model and the power-law model in the position space, which enables us to understand those in the wavenumber space presented in the main text. Since spatially averaged baryon asymmetry (B.7) is independent of the choice of $S(q)$, no difference between the delta-function model and the power-law model appears in the constraint from the overproduction of baryon asymmetry. However, as to the constraint of deuterium overproduction, Fig. 2 shows a trend of weaker constraint for the power-law model compared to the delta-function model. The origin of this trend is not so trivial, because the four-point function is not linear in the power spectrum and thus Fourier modes are not independent of each other. An intuitive interpretation is can be given as follows. The deltafunction model has a perfect periodicity and thus non-vanishing long-range correlation in the position space. If we compare asymptotic behavior of $\mathcal{G}(R)$ in $R \rightarrow \infty$ limit, for the deltafunction model, from (B.26), we find $\mathcal{G} \sim R^{-2}$ when $R \rightarrow \infty$. On the other hand for the power-law model, from (B.30), we find $\mathcal{G} \sim R^{-8-2 \alpha}$ when $R \rightarrow \infty$. Since our treatment is 


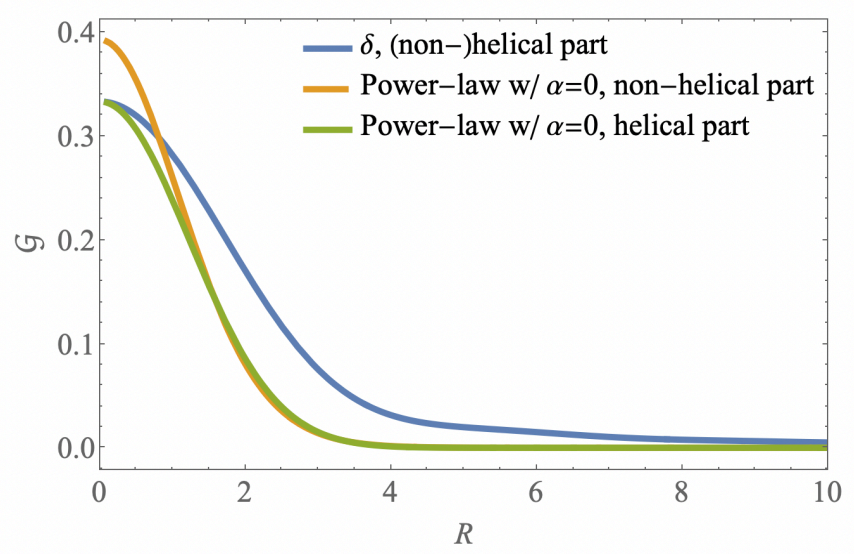

Figure 4. Comparison of the shapes of $\mathcal{G}(R)$ with the delta-function model and the power-law model with $\alpha=0$ is shown. $\mathcal{G}(R)$ has terms proportional to $1 / \epsilon^{2}$ originated from the non-helical part of the spectrum, and terms independent of $\epsilon$ originated from the helical part. In the delta-function model, the correlation function is exactly proportional to $1+1 / \epsilon^{2}$.

valid only for $\alpha>-3$, the tail in the large $R$ limit in the power-law model is more suppressed than that in the delta-function model, as can also be seen in Fig. 4. Since the neutron diffusion effect erases the small scale correlation, constraints on the models with stronger large-scale tail, that is, the delta-function model, are severer than that of the power-law model, which explains the tendency in Fig. 2.

\section{References}

[1] N. Aghanim et al. [Planck], "Planck 2018 results. VI. Cosmological parameters," Astron. Astrophys. 641 (2020), A6 [arXiv:1807.06209 [astro-ph.CO]].

[2] B. D. Fields, K. A. Olive, T. H. Yeh and C. Young, "Big-Bang Nucleosynthesis after Planck," JCAP 03 (2020), 010 [erratum: JCAP 11 (2020), E02] [arXiv:1912.01132 [astro-ph.CO]].

[3] A. D. Sakharov, "Violation of CP Invariance, C asymmetry, and baryon asymmetry of the universe," Sov. Phys. Usp. 34 (1991) no.5, 392-393

[4] V. A. Kuzmin, V. A. Rubakov and M. E. Shaposhnikov, "On the Anomalous Electroweak Baryon Number Nonconservation in the Early Universe," Phys. Lett. B 155 (1985), 36

[5] M. Fukugita and T. Yanagida, "Baryogenesis Without Grand Unification," Phys. Lett. B 174 (1986), 45-47

[6] M. Giovannini and M. E. Shaposhnikov, "Primordial magnetic fields, anomalous isocurvature fluctuations and big bang nucleosynthesis," Phys. Rev. Lett. 80 (1998), 22-25 [arXiv:hep-ph/9708303 [hep-ph]].

[7] M. Giovannini and M. E. Shaposhnikov, "Primordial hypermagnetic fields and triangle anomaly," Phys. Rev. D 57 (1998), 2186-2206 [arXiv:hep-ph/9710234 [hep-ph]]. 
[8] K. Bamba, "Baryon asymmetry from hypermagnetic helicity in dilaton hypercharge electromagnetism," Phys. Rev. D 74, 123504 (2006) [arXiv:hep-ph/0611152 [hep-ph]].

[9] T. Fujita and K. Kamada, "Large-scale magnetic fields can explain the baryon asymmetry of the Universe," Phys. Rev. D 93 (2016) no.8, 083520 [arXiv:1602.02109 [hep-ph]].

[10] K. Kamada and A. J. Long, "Baryogenesis from decaying magnetic helicity," Phys. Rev. D 94 (2016) no.6, 063501 [arXiv:1606.08891 [astro-ph.CO]].

[11] K. Kamada and A. J. Long, "Evolution of the Baryon Asymmetry through the Electroweak Crossover in the Presence of a Helical Magnetic Field," Phys. Rev. D 94 (2016) no.12, 123509 [arXiv:1610.03074 [hep-ph]].

[12] S. L. Adler, "Axial vector vertex in spinor electrodynamics," Phys. Rev. 177 (1969), 2426-2438

[13] J. S. Bell and R. Jackiw, "A PCAC puzzle: $\pi^{0} \rightarrow \gamma \gamma$ in the $\sigma$ model," Nuovo Cim. A 60 (1969), $47-61$

[14] G. 't Hooft, "Symmetry Breaking Through Bell-Jackiw Anomalies," Phys. Rev. Lett. 37 (1976), 8-11

[15] M. S. Turner and L. M. Widrow, "Inflation Produced, Large Scale Magnetic Fields," Phys. Rev. D 37 (1988), 2743

[16] W. D. Garretson, G. B. Field and S. M. Carroll, "Primordial magnetic fields from pseudoGoldstone bosons," Phys. Rev. D 46 (1992), 5346-5351 [arXiv:hep-ph/9209238 [hep-ph]].

[17] M. M. Anber and L. Sorbo, "N-flationary magnetic fields," JCAP 10, 018 (2006) [arXiv:astro-ph/0606534 [astro-ph]].

[18] M. Yoshimura, "Unified Gauge Theories and the Baryon Number of the Universe," Phys. Rev. Lett. 41, 281-284 (1978)

[19] A. Y. Ignatiev, N. V. Krasnikov, V. A. Kuzmin and A. N. Tavkhelidze, "Universal CP Noninvariant Superweak Interaction and Baryon Asymmetry of the Universe," Phys. Lett. B 76, 436-438 (1978)

[20] S. Weinberg, "Cosmological Production of Baryons," Phys. Rev. Lett. 42, 850-853 (1979)

[21] M. Joyce and M. E. Shaposhnikov, "Primordial magnetic fields, right-handed electrons, and the Abelian anomaly," Phys. Rev. Lett. 79, 1193-1196 (1997) [arXiv:astro-ph/9703005 [astro-ph]].

[22] H. Tashiro, T. Vachaspati and A. Vilenkin, "Chiral Effects and Cosmic Magnetic Fields," Phys. Rev. D 86, 105033 (2012) [arXiv:1206.5549 [astro-ph.CO]].

[23] Y. Akamatsu and N. Yamamoto, "Chiral Plasma Instabilities," Phys. Rev. Lett. 111, 052002 (2013) [arXiv:1302.2125 [nucl-th]].

[24] I. Affleck and M. Dine, "A New Mechanism for Baryogenesis," Nucl. Phys. B 249, 361-380 (1985)

[25] M. Dine, L. Randall and S. D. Thomas, "Baryogenesis from flat directions of the supersymmetric standard model," Nucl. Phys. B 458, 291-326 (1996) [arXiv:hep-ph/9507453 [hep-ph]].

[26] M. M. Anber and E. Sabancilar, "Hypermagnetic Fields and Baryon Asymmetry from Pseudoscalar Inflation," Phys. Rev. D 92, no.10, 101501 (2015) [arXiv:1507.00744 [hep-th]].

[27] P. Adshead, J. T. Giblin, T. R. Scully and E. I. Sfakianakis, "Magnetogenesis from axion inflation," JCAP 10, 039 (2016) [arXiv:1606.08474 [astro-ph.CO]].

[28] D. Jiménez, K. Kamada, K. Schmitz and X. J. Xu, "Baryon asymmetry and gravitational waves from pseudoscalar inflation," JCAP 12, 011 (2017) [arXiv:1707.07943 [hep-ph]].

[29] V. Domcke and K. Mukaida, "Gauge Field and Fermion Production during Axion Inflation," JCAP 11, 020 (2018) [arXiv:1806.08769 [hep-ph]]. 
[30] V. Domcke, B. von Harling, E. Morgante and K. Mukaida, "Baryogenesis from axion inflation," JCAP 10, 032 (2019) [arXiv:1905.13318 [hep-ph]].

[31] N. D. Barrie, "Big Bounce Baryogenesis," JCAP 08, 008 (2020) [arXiv:2001.04773 [hep-ph]].

[32] K. Kamada, "Return of grand unified theory baryogenesis: Source of helical hypermagnetic fields for the baryon asymmetry of the universe," Phys. Rev. D 97, no.10, 103506 (2018) [arXiv:1802.03055 [hep-ph]].

[33] K. Kamada and C. S. Shin, "Magnetogenesis from a rotating scalar: à la scalar chiral magnetic effect," JHEP 04, 185 (2020) [arXiv:1905.06966 [hep-ph]].

[34] M. D'Onofrio, K. Rummukainen and A. Tranberg, "Sphaleron Rate in the Minimal Standard Model," Phys. Rev. Lett. 113, no.14, 141602 (2014) [arXiv:1404.3565 [hep-ph]].

[35] M. D'Onofrio and K. Rummukainen, "Standard model cross-over on the lattice," Phys. Rev. D 93, no.2, 025003 (2016) [arXiv:1508.07161 [hep-ph]].

[36] A. Neronov and I. Vovk, "Evidence for strong extragalactic magnetic fields from Fermi observations of TeV blazars," Science 328 (2010), 73-75 [arXiv:1006.3504 [astro-ph.HE]].

[37] F. Tavecchio, G. Ghisellini, L. Foschini, G. Bonnoli, G. Ghirlanda and P. Coppi, "The intergalactic magnetic field constrained by Fermi/LAT observations of the TeV blazar 1ES 0229+200," Mon. Not. Roy. Astron. Soc. 406, L70-L74 (2010) [arXiv:1004.1329 [astro-ph.CO]].

[38] S. Ando and A. Kusenko, "Evidence for Gamma-Ray Halos Around Active Galactic Nuclei and the First Measurement of Intergalactic Magnetic Fields," Astrophys. J. Lett. 722, L39 (2010) [arXiv:1005.1924 [astro-ph.HE]].

[39] K. Dolag, M. Kachelriess, S. Ostapchenko and R. Tomas, "Lower limit on the strength and filling factor of extragalactic magnetic fields," Astrophys. J. Lett. 727, L4 (2011) [arXiv:1009.1782 [astro-ph.HE]].

[40] W. Essey, S. Ando and A. Kusenko, "Determination of intergalactic magnetic fields from gamma ray data," Astropart. Phys. 35, 135-139 (2011) [arXiv:1012.5313 [astro-ph.HE]].

[41] A. M. Taylor, I. Vovk and A. Neronov, "Extragalactic magnetic fields constraints from simultaneous GeV-TeV observations of blazars," Astron. Astrophys. 529, A144 (2011) [arXiv:1101.0932 [astro-ph.HE]].

[42] K. Takahashi, M. Mori, K. Ichiki, S. Inoue and H. Takami, "Lower Bounds on Magnetic Fields in Intergalactic Voids from Long-term GeV-TeV Light Curves of the Blazar Mrk 421," Astrophys. J. Lett. 771, L42 (2013) [arXiv:1303.3069 [astro-ph.CO]].

[43] J. D. Finke, L. C. Reyes, M. Georganopoulos, K. Reynolds, M. Ajello, S. J. Fegan and K. McCann, "Constraints on the Intergalactic Magnetic Field with Gamma-Ray Observations of Blazars," Astrophys. J. 814 (2015) no.1, 20 [arXiv:1510.02485 [astro-ph.HE]].

[44] M. Ackermann et al. [Fermi-LAT], "The Search for Spatial Extension in High-latitude Sources Detected by the Fermi Large Area Telescope," Astrophys. J. Suppl. 237 (2018) no.2, 32 [arXiv:1804.08035 [astro-ph.HE]].

[45] R. Alves Batista and A. Saveliev, "Multimessenger Constraints on Intergalactic Magnetic Fields from the Flare of TXS 0506+056," Astrophys. J. Lett. 902 (2020) no.1, L11 [arXiv:2009.12161 [astro-ph.HE]].

[46] Y. Akrami et al. [Planck], "Planck 2018 results. X. Constraints on inflation," Astron. Astrophys. 641 (2020), A10 [arXiv:1807.06211 [astro-ph.CO]].

[47] M. Kawasaki, T. Sekiguchi and T. Takahashi, "Differentiating CDM and Baryon Isocurvature Models with $21 \mathrm{~cm}$ Fluctuations," JCAP 10 (2011), 028 [arXiv:1104.5591 [astro-ph.CO]]. 
[48] Y. Takeuchi and S. Chongchitnan, "Constraining isocurvature perturbations with the $21 \mathrm{~cm}$ emission from minihaloes," Mon. Not. Roy. Astron. Soc. 439 (2014) no.1, 1125-1135 [arXiv:1311.2585 [astro-ph.CO]].

[49] J. H. Applegate, C. J. Hogan and R. J. Scherrer, "Cosmological Baryon Diffusion and Nucleosynthesis," Phys. Rev. D 35, 1151-1160 (1987)

[50] C. Alcock, G. M. Fuller and G. J. Mathews, "The Quark - hadron phase transition and primordial nucleosynthesis," Astrophys. J. 320, 439-447 (1987)

[51] K. Inomata, M. Kawasaki, A. Kusenko and L. Yang, "Big Bang Nucleosynthesis Constraint on Baryonic Isocurvature Perturbations," JCAP 12 (2018), 003 [arXiv:1806.00123 [astro-ph.CO]].

[52] R. Banerjee and K. Jedamzik, "The Evolution of cosmic magnetic fields: From the very early universe, to recombination, to the present," Phys. Rev. D 70 (2004), 123003 [arXiv:astro-ph/0410032 [astro-ph]].

[53] G. Baym and H. Heiselberg, "The Electrical conductivity in the early universe," Phys. Rev. D 56 (1997), 5254-5259 [arXiv:astro-ph/9704214 [astro-ph]].

[54] P. B. Arnold, G. D. Moore and L. G. Yaffe, "Transport coefficients in high temperature gauge theories. 1. Leading log results," JHEP 11 (2000), 001 [arXiv:hep-ph/0010177 [hep-ph]].

[55] K. Kajantie, M. Laine, K. Rummukainen and M. E. Shaposhnikov, "A Nonperturbative analysis of the finite T phase transition in SU(2) x U(1) electroweak theory," Nucl. Phys. B 493 (1997), 413-438 [arXiv:hep-lat/9612006 [hep-lat]].

[56] B. Ratra, "Cosmological 'seed' magnetic field from inflation," Astrophys. J. Lett. 391 (1992), L1-L4 doi:10.1086/186384

[57] K. Bamba and J. Yokoyama, "Large scale magnetic fields from inflation in dilaton electromagnetism," Phys. Rev. D 69 (2004), 043507 [arXiv:astro-ph/0310824 [astro-ph]].

[58] J. Martin and J. Yokoyama, "Generation of Large-Scale Magnetic Fields in Single-Field Inflation," JCAP 01 (2008), 025 [arXiv:0711.4307 [astro-ph]].

[59] C. J. Hogan, "Magnetohydrodynamic Effects of a First-Order Cosmological Phase Transition," Phys. Rev. Lett. 51 (1983), 1488-1491

[60] J. M. Quashnock, A. Loeb and D. N. Spergel, "Magnetic Field Generation During the Cosmological QCD Phase Transition," Astrophys. J. Lett. 344 (1989), L49-L51

[61] T. Vachaspati, "Magnetic fields from cosmological phase transitions," Phys. Lett. B 265 (1991), $258-261$

[62] H. K. Moffatt, Magnetic field generation in electrically conducting fluids, Cambridge University Press, Cambridge, 1978

[63] W. Hu and N. Sugiyama, "Thermal history constraints on the isocurvature baryon model," Astrophys. J. 436 (1994), 456-466 [arXiv:astro-ph/9403031 [astro-ph]].

[64] J. B. Dent, D. A. Easson and H. Tashiro, "Cosmological constraints from CMB distortion," Phys. Rev. D 86 (2012), 023514 [arXiv:1202.6066 [astro-ph.CO]].

[65] M. Kawasaki and T. Sekiguchi, "Cosmological Constraints on Isocurvature and Tensor Perturbations," Prog. Theor. Phys. 120 (2008), 995-1016 [arXiv:0705.2853 [astro-ph]].

[66] M. Kawasaki, T. Sekiguchi, T. Takahashi and S. Yokoyama, "Isocurvature perturbations and tensor mode in light of Planck and BICEP2," JCAP 08 (2014), 043 [arXiv:1404.2175 [astro-ph.CO]].

[67] T. Sekiguchi, H. Tashiro, J. Silk and N. Sugiyama, "Cosmological signatures of tilted isocurvature perturbations: reionization and 21cm fluctuations," JCAP 03 (2014), 001 [arXiv:1311.3294 [astro-ph.CO]]. 
[68] G. J. Mathews, et al., "Coupled baryon diffusion and nucleosynthesis in the early universe," Astrophys. J. 358 (1990), 36-46

[69] K. Kainulainen, H. Kurki-Suonio and E. Sihvola, "Inhomogeneous big bang nucleosynthesis in light of recent observations," Phys. Rev. D 59 (1999), 083505 [arXiv:astro-ph/9807098 [astro-ph]].

[70] J. F. Lara, "Neutron diffusion and nucleosynthesis in an inhomogeneous Big Bang model," Phys. Rev. D 72 (2005), 023509 [arXiv:astro-ph/0506364 [astro-ph]].

[71] J. F. Lara, T. Kajino and G. J. Mathews, "Inhomogeneous big bang nucleosynthesis revisited," Phys. Rev. D 73 (2006), 083501 [arXiv:astro-ph/0603817 [astro-ph]].

[72] S. Matsuura, S. I. Fujimoto, S. Nishimura, M. A. Hashimoto and K. Sato, "Heavy element production in inhomogeneous big bang nucleosynthesis," Phys. Rev. D 72 (2005), 123505 [arXiv:astro-ph/0507439 [astro-ph]].

[73] D. Grasso and H. R. Rubinstein, "Magnetic fields in the early universe," Phys. Rept. 348 (2001), 163-266 [arXiv:astro-ph/0009061 [astro-ph]].

[74] R. Durrer and A. Neronov, "Cosmological Magnetic Fields: Their Generation, Evolution and Observation," Astron. Astrophys. Rev. 21 (2013), 62 doi:10.1007/s00159-013-0062-7 [arXiv:1303.7121 [astro-ph.CO]].

[75] A. Brandenburg and T. Kahniashvili, "Classes of hydrodynamic and magnetohydrodynamic turbulent decay," Phys. Rev. Lett. 118 (2017) no.5, 055102 [arXiv:1607.01360 [physics.flu-dyn]].

[76] P. Olesen, "On inverse cascades in astrophysics," Phys. Lett. B 398 (1997), 321-325 [arXiv:astro-ph/9610154 [astro-ph]].

[77] A. Brandenburg, T. Kahniashvili, S. Mandal, A. Roper Pol, A. G. Tevzadze and T. Vachaspati, "Evolution of hydromagnetic turbulence from the electroweak phase transition," Phys. Rev. D 96 (2017) no.12, 123528 [arXiv:1711.03804 [astro-ph.CO]].

[78] S. Candelaresi and A. Brandenburg, "Decay of helical and non-helical magnetic knots," Phys. Rev. E 84 (2011), 016406 [arXiv:1103.3518 [astro-ph.SR]].

[79] P. A. R. Ade et al. [Planck], "Planck 2015 results. XIX. Constraints on primordial magnetic fields," Astron. Astrophys. 594 (2016), A19 [arXiv:1502.01594 [astro-ph.CO]].

[80] J. D. Barrow, P. G. Ferreira and J. Silk, "Constraints on a primordial magnetic field," Phys. Rev. Lett. 78 (1997), 3610-3613 [arXiv:astro-ph/9701063 [astro-ph]].

[81] P. Blasi, S. Burles and A. V. Olinto, "Cosmological magnetic fields limits in an inhomogeneous universe," Astrophys. J. Lett. 514 (1999), L79-L82 [arXiv:astro-ph/9812487 [astro-ph]].

[82] K. Jedamzik, V. Katalinic and A. V. Olinto, "A Limit on primordial small scale magnetic fields from CMB distortions," Phys. Rev. Lett. 85 (2000), 700-703 [arXiv:astro-ph/9911100 [astro-ph]].

[83] R. Durrer, P. G. Ferreira and T. Kahniashvili, "Tensor microwave anisotropies from a stochastic magnetic field," Phys. Rev. D 61 (2000), 043001 [arXiv:astro-ph/9911040 [astro-ph]].

[84] D. G. Yamazaki, T. Kajino, G. J. Mathew and K. Ichiki, "The Search for a Primordial Magnetic Field," Phys. Rept. 517 (2012), 141-167 [arXiv:1204.3669 [astro-ph.CO]].

[85] P. Trivedi, K. Subramanian and T. R. Seshadri, "Primordial Magnetic Field Limits from Cosmic Microwave Background Bispectrum of Magnetic Passive Scalar Modes," Phys. Rev. D 82 (2010), 123006 [arXiv:1009.2724 [astro-ph.CO]].

[86] D. Paoletti and F. Finelli, "CMB Constraints on a Stochastic Background of Primordial Magnetic Fields," Phys. Rev. D 83 (2011), 123533 [arXiv:1005.0148 [astro-ph.CO]].

[87] J. R. Shaw and A. Lewis, "Massive Neutrinos and Magnetic Fields in the Early Universe," Phys. Rev. D 81 (2010), 043517 [arXiv:0911.2714 [astro-ph.CO]]. 
[88] J. R. Shaw and A. Lewis, "Constraining Primordial Magnetism," Phys. Rev. D 86 (2012), 043510 [arXiv:1006.4242 [astro-ph.CO]l.

[89] A. Brandenburg, R. Durrer, T. Kahniashvili, S. Mandal and W. W. Yin, "Statistical Properties of Scale-Invariant Helical Magnetic Fields and Applications to Cosmology," JCAP 08 (2018), 034 [arXiv:1804.01177 [astro-ph.CO]].

[90] A. C. Davis, M. Lilley and O. Tornkvist, "Relaxing the bounds on primordial magnetic seed fields," Phys. Rev. D 60 (1999), 021301 [arXiv:astro-ph/9904022 [astro-ph]].

[91] A. E. Broderick, P. Chang and C. Pfrommer, "The Cosmological Impact of Luminous TeV Blazars I: Implications of Plasma Instabilities for the Intergalactic Magnetic Field and Extragalactic Gamma-Ray Background," Astrophys. J. 752 (2012), 22 [arXiv:1106.5494 [astro-ph.CO]]. 